Série des Documents de Travail

\author{
$n^{\circ}$ 2012-03 \\ Shock on Variable or Shock on \\ Distribution with Application \\ to Stress-Tests \\ S. DUBECQ ${ }^{1}$ \\ C. GOURIEROUX ${ }^{2}$
}

February 2012

The second author gratefully acknowledges financial support of NSERC, Canada, and of the Chair AXA/Risk Foundation : "Large Risks in Insurance". The views expressed in this paper are those of the authors and do not necessarily reflect those of the Banque de France. We thank Jean-Paul Laurent, Martin Schweizer, and the participants at the Banque de France and CREST internal seminars for helpful comments, and J.P. Renne, B. Saes-Escorbiac, and A. Touchais for kindly providing us with the sovereign bond data.

Les documents de travail ne reflètent pas la position du CREST et n'engagent que leurs auteurs. Working papers do not reflect the position of CREST but only the views of the authors.

\footnotetext{
1 Banque de France and CREST. simon.dubecq@banque-france.fr

2 CREST and University of Toronto (Canada). christian.gourieroux@ensae.fr
} 


\title{
Shock on Variable or Shock on Distribution with Application to Stress-Tests
}

\begin{abstract}
The shocks on a stochastic system can be defined by means of either distribution, or variable. We relate these approaches and provide the link between the global and local effects of both types of shocks. These methodologies are used to perform stress-tests on the portfolio of financial institutions by means of shocks on systematic factors, for which we distinguish the cases of crystallized and optimally updated portfolios. The approach is illustrated by an analysis of the risk of sovereign bonds of the Eurozone.
\end{abstract}

Keywords : Shock, Copula, Extreme Risk, Stress-Test, Factor Model, Systemic Risk, Portfolio Management, Sovereign Bonds. 


\section{Introduction}

The comparison of risks or the analysis of the effects of shocks on a risky portfolio value are problems concerning a comparison of two distributions. However, these questions are often presented in the economic and finance literature in terms of stochastic variables. This is an abuse of language. It is likely introduced to facilitate the understanding of the notion by the standard reader, but it can also imply misleading interpretations and errors in implementing the notion.

To illustrate this practice, let us consider the notion of second-order stochastic dominance. Rothschild, Stiglitz (1970), Theorem 2, have proposed three equivalent characterizations of this notion. Loosely speaking they consider two variables $Y_{0}, Y_{1}$ with respective cumulative distributions $F_{0}$ and $F_{1}$. For expository purpose, let us interpret $Y_{0}$ and $Y_{1}$ as the prices of two assets 0 and 1, or equivalently, as the value of two portfolios completely invested in asset 0 and 1 , respectively. The investment $Y_{0}$ in asset 0 dominates the investment $Y_{1}$ in asset 1 at the second-order if one of the following equivalent conditions i)-iii) is satisfied :

i) $\int_{0}^{K}\left[1-F_{0}(y)\right] d y \geq \int_{0}^{K}\left[1-F_{1}(y)\right] d y, \forall K$.

Let us consider the price, under the historical distribution ${ }^{3}$, of a European call option written on the investment $Y_{0}$ with strike $K$, that is, the price of a derivative with payoff equal to $Y_{0}-K$, if $Y_{0} \geq K$, equal to 0 , otherwise. We have :

$$
\begin{aligned}
\mathbb{E}\left(\left[Y_{0}-K\right]^{+}\right) & =\int_{K}^{+\infty}(y-K) f_{0}(y) d y \\
& =-\int_{K}^{+\infty}(y-K) d\left[1-F_{0}(y)\right] \\
& =-\left[(y-K)\left(1-F_{0}(y)\right)\right]_{K}^{+\infty}+\int_{K}^{+\infty}\left[1-F_{0}(y)\right] d y \\
& =\int_{K}^{+\infty}\left[1-F_{0}(y)\right] d y \\
& =E\left(Y_{0}\right)-\int_{0}^{K}\left[1-F_{0}(y)\right] d y, \text { since } Y_{0}>0 .
\end{aligned}
$$

Thus, condition i) means that, for a given strike $K$, a European call option contract written on the price of asset 0 is always cheaper than the similar contract written on asset

\footnotetext{
${ }^{3}$ Equivalently, this is the price for a risk-neutral investor.
} 
1, whenever $Y_{0}$ and $Y_{1}$ have the same mean. Therefore, the investment $Y_{0}$ dominates $Y_{1}$ under i), since the price of the insurance against a drop in investment's value is lower for asset 0 .

ii) $\mathbb{E}\left[U\left(Y_{0}\right)\right] \geq \mathbb{E}\left[U\left(Y_{1}\right)\right]$, for any bounded concave function $U$.

Condition ii) means that any risk-averse agent, i.e. with concave utility function, would prefer asset 0 to asset 1 .

iii) There exists a variable $Z$ such that : $Y_{1}=Y_{0}+Z$, with $\mathbb{E}\left[Z \mid Y_{0}\right]=0$.

The first and second characterizations show clearly that the concept of stochastic dominance concerns the distributions. The third characterization seems to be of a different type. It says that we can pass from $Y_{0}$ to $Y_{1}$ by adding a stochastic shock with zero conditional mean. It concerns variables themselves, but on an extended space, since the comparison of the marginal distributions of $Y_{0}$ and $Y_{1}$ implicit in i) and ii) is now replaced by a condition, which involves the joint distribution of $\left(Y_{0}, Y_{1}\right)$ on the product space. As seen from the proof in Rothschild, Stiglitz (1970), the third characterization can only be obtained after having constructed an artificial product space [see also Strassen (1965), Armbruster (2011)].

In Section 2, we consider a parametric family of cumulative distribution functions $\left(F_{\delta}, \delta \in I\right)$, and we show that they can always be considered as the marginal distributions of a family of variable $Y_{\delta}$ defined by a stochastic equation $Y_{\delta}=h\left(Y_{0}, \varepsilon ; \delta\right)$, where $\varepsilon$ is a variable independent of $Y_{0}$. As in Rothschild, Stiglitz, the result requires the construction of an artificial product space. The "equivalent" representation of the family of distributions allows to understand why shocks can be defined in terms of either parameter, or distribution, or variable.

The effect of a small change of $\delta$ on the distribution is usually studied by considering a Taylor expansion of distribution $F_{\delta}$ with respect to $\delta$. In Section 3 we show how an equivalent expansion can be performed in terms of variables and relate the two types of expansions. Some examples of specification of shocks are given in Section 4. While common practices usually perform stress-testing exercises by considering deterministic shock on crystallized portfolio value, we show that our results allow for much richer exercises. 
As an illustration, the stress-test methodology is applied in Section 5 to portfolios of sovereign bonds. We consider sovereign bonds for different countries on period 2001-2011, and extract the underlying factors by a principal component analysis. The distribution of these factors on periods 2001-2007 and 2007-2011 shows a significant change due to the recent financial crisis. Then we consider period 2001-2007 as a benchmark and shock the first factor by contaminating the benchmark distribution with crisis specific distribution. We analyze the effects of this contamination on a crystallized portfolio and an optimally updated portfolio, both for shocks in distribution and in variable. Section 6 concludes. The technical proofs are gathered in Appendices. 


\section{Family of Distributions or Family of Variables}

The aim of this section is to relate a parametric modeling written in terms of distributions and a parametric modeling written in terms of stochastic variables. For expository purpose, we consider continuous distributions on $\mathbb{R}$ and a scalar parameter $\delta$ (the extension to multivariate distributions is given in Appendix 1 i)).

The first type of modeling defines a family of distributions $\left\{F_{\delta}, \delta \in I\right\}$, where $I$ denotes an interval of $\mathbb{R}$ and $F_{\delta}$ the cumulative distribution function. The second modeling is based on some relationship between variables : $Y_{\delta}=h\left(Y_{0}, \varepsilon ; \delta\right), \delta \in I$, say, where $Y_{0}$ and $\varepsilon$ are independent variables with $Y_{0}$ following $F_{0}$ and $\varepsilon$ following the uniform distribution on $[0,1]$. The questions solved in this section are the following ones :

i) Given the parametric family $\left\{F_{\delta}, \delta \in I\right\}$, is it always possible to find a variable $\varepsilon$ and a function $h$ such that the marginal distribution of $Y_{\delta}$ is $F_{\delta}$, for any $\delta \in I$ ?

ii) Is such a function $h$ unique, if it exists?

\subsection{Copula}

Whereas the modeling in distribution involves the one-dimensional space $(\mathbb{R}, \mathbb{B}(\mathbb{R}))$, the modeling in variable involves a two dimensional space $\left(\mathbb{R}^{2}, \mathbb{B}\left(\mathbb{R}^{2}\right)\right)$. Thus, we have first to introduce such a bidimensional artificial space. Let us denote by $(U, V)$ a pair of variables on this space with marginal distributions which are uniform on $[0,1]$, and a joint c.d.f. $C(u, v)=\mathbb{P}[U<u, V<v], u, v \in[0,1]$. The variables are usually called rank variables and function $C$ is the copula cumulative function.

Let us first consider two values, 0 and 1 , say, of parameter $\delta$, and the associated distributions $F_{0}$ and $F_{1}$. By Sklar theorem [Sklar (1959)], the variables:

$$
Y_{0}=F_{0}^{-1}(U), Y_{1}=F_{1}^{-1}(V),
$$

have the joint c.d.f. :

$$
\mathbb{P}\left[Y_{0}<y_{0}, Y_{1}<y_{1}\right]=C\left[F_{0}\left(y_{0}\right), F_{1}\left(y_{1}\right)\right] .
$$

In particular the marginal distribution of $Y_{0}$ (resp. $\left.Y_{1}\right)$ is $F_{0}$ (resp. $\left.F_{1}\right)$. 
Let us now consider the conditional c.d.f. of $Y_{1}$ given $Y_{0}$. We have [see Joe (1997), p.245] :

$$
F_{1 \mid 0}\left(y_{1} \mid Y_{0}\right) \equiv \mathbb{P}\left[Y_{1}<y_{1} \mid Y_{0}\right]=\frac{\partial C}{\partial u}\left[F_{0}\left(Y_{0}\right), F_{1}\left(y_{1}\right)\right]
$$

Thus, by using the inverse transform method, the variable :

$$
\varepsilon=F_{1 \mid 0}\left(Y_{1} \mid Y_{0}\right)=\frac{\partial C}{\partial u}\left[F_{0}\left(Y_{0}\right), F_{1}\left(Y_{1}\right)\right]
$$

follows a uniform distribution on $[0,1]$ and is independent of $Y_{0}$. We deduce the expected expression in terms of variables :

$$
\begin{aligned}
Y_{1} & =\left(F_{1 \mid 0}\left(\bullet \mid Y_{0}\right)\right)^{-1}(\varepsilon) \\
& =\left(\frac{\partial C}{\partial u}\left[F_{0}\left(Y_{0}\right), F_{1}(\bullet)\right]\right)^{-1}(\varepsilon) \\
& =h\left(Y_{0}, \varepsilon ; 1\right), \text { say. }
\end{aligned}
$$

Thus we have shown the existence of function $h$. Moreover, by increasing the dimension of the space, we allow for a variety of choices of copula $C$ and then of function $h$. Finally, equation (2.4) can also be written as :

$$
\varepsilon=\frac{\partial C}{\partial u}(U, V),
$$

which shows how the uniform variable $\varepsilon$ depends on the basic uniform variables $U$ and $V$ in a complicated nonlinear way.

\subsection{Extension to families}

The result of Section 2.1 can be applied to any pair of parameter values $(0, \delta)$, associated distributions $\left(F_{0}, F_{\delta}\right)$, and variables $\left(Y_{0}, Y_{\delta}\right)$. We get :

$$
\varepsilon_{\delta}=\frac{\partial C_{\delta}}{\partial u}\left[F_{0}\left(Y_{0}\right), F_{\delta}\left(Y_{\delta}\right)\right], \delta \in(0,1)
$$

and

$$
Y_{\delta}=\left(\frac{\partial C_{\delta}}{\partial u}\left[F_{0}\left(Y_{0}\right), F_{\delta}(\bullet)\right]\right)^{-1}\left(\varepsilon_{\delta}\right), \delta \in(0,1),
$$

where the copula is now indexed by parameter $\delta$. All variables $\varepsilon_{\delta}$ are uniformly distributed on $[0,1]$ and independent of $Y_{0}$. In practice the shock defined in terms of variable involves 
an innovation $\varepsilon$, which is independent of $\delta$. This is easily derived if we consider equality in distribution. Indeed, equation (2.6) implies the equality in distribution

$$
Y_{\delta} \stackrel{d}{=} h\left(Y_{0}, \varepsilon ; \delta\right), \delta \in[0,1]
$$

where

$$
h\left(Y_{0}, \varepsilon ; \delta\right)=\left(\frac{\partial C_{\delta}}{\partial u}\left[F_{0}\left(Y_{0}\right), F_{\delta}(\bullet)\right]\right)^{-1}(\varepsilon),
$$

$\varepsilon$ is independent of $\delta$, of $Y_{0}$, and uniformly distributed on $[0,1]$.

\subsection{Shocks}

Let us now discuss the introduction of shocks.

i) The shock can be defined by means of parameter $\delta$. For instance, the parameter can pass from value 0 to value $\delta$, say.

ii) The corresponding effect on distribution will be the change from $F_{0}$ to $F_{\delta}$.

iii) Let us finally consider the variable interpretation. Equality (2.7) in distribution has to be replaced by an equality in terms of variables. In fact, we can define

$$
Y_{\delta}=h\left(Y_{0}, \varepsilon ; \delta\right),
$$

with $h$ satisfying (2.8), whenever the following coherency condition is satisfied :

$$
Y_{0}=h\left(Y_{0}, \varepsilon ; 0\right)
$$

Then the shock on variables is $Y_{\delta}-Y_{0}=h\left(Y_{0}, \varepsilon ; \delta\right)-Y_{0}$. For a given $Y_{0}$, this is in general a stochastic shock due to the effect of the uniform stochastic variable $\varepsilon$.

The coherency condition (2.9) implies restrictions on the choice of copula in (2.8). Let us for instance consider the Gaussian copula with correlation parameter $\rho(\delta)$, and variables $Y_{0}, Y_{\delta}$, whose marginal distributions are $F(y ; 0), F(y ; \delta)$, respectively. We have (see Appendix 4 i)) :

$$
\varepsilon=\Phi\left(\frac{\Phi^{-1}\left[F\left(Y_{\delta} ; \delta\right)\right]-\rho(\delta) \Phi^{-1}\left[F\left(Y_{0} ; 0\right)\right]}{\sqrt{1-\rho^{2}(\delta)}}\right)
$$


where $\Phi$ is the standard Gaussian c.d.f. Thus :

$$
\Phi^{-1}\left[F\left(Y_{\delta} ; \delta\right)\right]=\rho(\delta) \Phi^{-1}\left[F\left(Y_{0} ; 0\right)\right]+\sqrt{1-\rho^{2}(\delta)} \Phi^{-1}(\varepsilon) .
$$

Equation 2.10 shows that the coherency condition restricts the Gaussian copula to be such that $\rho(0)=1$.

Finally note that the definition of the direction of the shock is more accurate with the specification in variable. Indeed, it specifies $Y_{\delta}-Y_{0}$ with respect to $Y_{0}$. Thus it requires the specification of the joint distribution of $Y_{0}$ and $Y_{\delta}$, whereas the specification in terms of distribution demands the unconditional distributions only. This explains why different specifications of shocks in terms of variable can lead to a same specification in terms of distribution. 


\section{Local Analysis}

We have seen in Section 2 how to link the approaches in distributions and in variables in a global analysis of shocks. However, extreme effects can result from small shocks when the system is nonlinear. In applications to finance, these nonlinearities are due to derivatives (call options, credit derivatives) included in the portfolio as well as the nonlinear portfolio management strategies. This explains why a global analysis has to be completed by a local analysis.

The effect of a small chang $€^{4}$ in $\delta$ is usually treated by considering appropriate Taylor expansions. These expansions can be done from the distributions themselves, or from the interpretation in terms of variables. We consider below these expansions in a neighborhood of $\delta=0$.

\subsection{Expansion of the distribution}

Let us denote $f(y ; \delta)$ the density function corresponding to $F_{\delta}$. Under standard regularity conditions, we get the Taylor expansion at order $p$ :

$$
f(y ; \delta)=f(y ; 0)+\sum_{j=1}^{p} \frac{\delta^{j}}{j !} \frac{\partial^{j} f(y ; 0)}{\partial \delta^{j}}+o\left(\delta^{p}\right),
$$

where $o($.$) denotes a deterministic negligible term.$

In particular, we get at second-order :

$$
\begin{aligned}
f(y ; \delta) & =f(y ; 0)+\delta \frac{\partial f(y ; 0)}{\partial \delta}+\frac{\delta^{2}}{2} \frac{\partial^{2} f(y ; 0)}{\partial \delta^{2}}+o\left(\delta^{2}\right) \\
& =f(y ; 0)\left\{1+\delta \frac{\partial \log f(y ; 0)}{\partial \delta}+\frac{\delta^{2}}{2}\left[\frac{\partial^{2} \log f(y ; 0)}{\partial \delta^{2}}+\left(\frac{\partial \log f(y ; 0)}{\partial \delta}\right)^{2}\right]\right\}+o\left(\delta^{2}\right),
\end{aligned}
$$

[see Chesher (1983), (1984) for an application of this expansion for testing neglected heterogeneity].

\footnotetext{
${ }^{4} \mathrm{~A}$ small change in $\delta$ implies a large change on variable $Y_{0}$, if the stochastic direction of the shock concerns extreme risks (see Section 5).
} 


\subsection{Expansion in terms of variable}

Let us now consider the model :

$$
Y_{\delta}=h\left(Y_{0}, \varepsilon ; \delta\right)
$$

We could apply a Taylor expansion at order $p$ to get :

$$
Y_{\delta}=Y_{0}+\sum_{j=1}^{p}\left(\frac{\delta^{j}}{j !} \frac{\partial^{j} h\left(Y_{0}, \varepsilon ; 0\right)}{\partial \delta^{j}}\right)+o_{\mathbb{P}}\left(\delta^{p}\right),
$$

where $o_{\mathbb{P}}($.$) denotes a negligible term in probability.$

However, such an expansion would be difficult to interpret in terms of distributions. Instead, we consider below the approximate computation of an expectation $\mathbb{E}\left[g\left(Y_{\delta}\right)\right]$, where $g$ is an infinitely differentiable function with compact support. We get :

$$
\begin{aligned}
\mathbb{E}\left[g\left(Y_{\delta}\right)\right] & =\mathbb{E}\left[g\left(h\left(Y_{0}, \varepsilon ; \delta\right)\right)\right] \\
& =\mathbb{E}\left[g\left(Y_{0}\right)\right]+\sum_{j=1}^{p}\left\{\frac{\delta^{j}}{j !} \mathbb{E}\left[\frac{\partial^{j}}{\partial \delta^{j}} g\left(h\left(Y_{0}, \varepsilon ; \delta\right)\right)\right]_{\delta=0}\right\}+o\left(\delta^{p}\right) .
\end{aligned}
$$

Then, we can apply Faa di Bruno's formula [see Faa di Bruno (1855), Johnson (2002), Spindler (2005)], which provides the $j$-th derivative of a composite function.

Lemma 1 : Faa di Bruno's formula

$$
\frac{d^{n}}{d t^{n}} g[h(t)]=\sum_{\operatorname{Dio}_{n}} \frac{n !}{k_{1} ! k_{2} ! \ldots k_{n} !} g^{(k)}[h(t)]\left(\frac{h^{(1)}(t)}{1 !}\right)^{k_{1}}\left(\frac{h^{(2)}(t)}{2 !}\right)^{k_{2}} \ldots\left(\frac{h^{(n)}(t)}{n !}\right)^{k_{n}}
$$

where $g^{(k)}$ denotes the $k^{\text {th }}$ derivative of function $g$, and where the sum is over all nonnegative integer solutions of the Diophantine equation : $k_{1}+2 k_{2}+\ldots+n k_{n}=n$, and where $k=k_{1}+\ldots+k_{n}$.

We deduce that :

$$
\mathbb{E}\left[g\left(Y_{\delta}\right)\right]=\mathbb{E}\left[g\left(Y_{0}\right)\right]+\sum_{j=1}^{p}\left\{\frac{\delta^{j}}{j !} \sum_{k=1}^{j} \mathbb{E}\left[g^{(k)}\left(Y_{0}\right) A_{j, k}\left(Y_{0}, \varepsilon\right)\right]\right\}+o\left(\delta^{p}\right),
$$


where

$$
A_{j, k}\left(Y_{0}, \varepsilon\right)=\sum_{\operatorname{Dio}_{j, k}}\left\{\frac{j !}{k_{1} ! k_{2} ! \ldots k_{j} !}\left(\frac{1}{1 !} \frac{\partial h\left(Y_{0}, \varepsilon, 0\right)}{\partial \delta}\right)^{k_{1}} \ldots\left(\frac{1}{j !} \frac{\partial^{j} h\left(Y_{0}, \varepsilon, 0\right)}{\partial \delta^{j}}\right)^{k_{j}}\right\},
$$

and the sum is over $k_{1}, \ldots, k_{j}$ such that $k_{1}+2 k_{2}+\ldots+j k_{j}=j$ and $k_{1}+\ldots+k_{j}=k$.

We can also write by the Iterated Expectation Theorem :

$$
\mathbb{E}\left[g\left(Y_{\delta}\right)\right]=\mathbb{E}\left[g\left(Y_{0}\right)\right]+\sum_{j=1}^{p}\left\{\frac{\delta^{j}}{j !} \sum_{k=1}^{j} \mathbb{E}\left[g^{(k)}\left(Y_{0}\right) a_{j, k}\left(Y_{0}\right)\right]\right\}+o\left(\delta^{p}\right),
$$

where

$$
a_{j, k}\left(Y_{0}\right)=\mathbb{E}\left[A_{j, k}\left(Y_{0}, \varepsilon\right) \mid Y_{0}\right]
$$

Finally the following Lemma is proved in Appendix 2.

Lemma 2 : We have

$$
\mathbb{E}\left[g^{(k)}\left(Y_{0}\right) a\left(Y_{0}\right)\right]=(-1)^{k} \mathbb{E}\left[\frac{g\left(Y_{0}\right)}{f\left(Y_{0} ; 0\right)} \frac{d^{k}}{d y^{k}}\left[a\left(Y_{0}\right) f\left(Y_{0} ; 0\right)\right]\right],
$$

for any $k$ and function $a$.

Thus, we can rewrite equation (3.4) as :

$$
\mathbb{E}\left[g\left(Y_{\delta}\right)\right]=\mathbb{E}\left[g\left(Y_{0}\right)\right]+\sum_{j=1}^{p}\left\{\frac{\delta^{j}}{j !} \sum_{k=1}^{j}(-1)^{k} \mathbb{E}\left[\frac{g\left(Y_{0}\right)}{f\left(Y_{0} ; 0\right)} \frac{d^{k}}{d y^{k}}\left[a_{j, k}\left(Y_{0}\right) f\left(Y_{0} ; 0\right)\right]\right]\right\}+o\left(\delta^{p}\right) .
$$

The knowledge of the expectation $\mathbb{E}\left[g\left(Y_{\delta}\right)\right]$ for all infinitely differentiable functions with compact support characterizes the distribution of $Y_{\delta}$. Thus, by comparing expansions (3.1) and (3.6), we see how the Taylor expansion in terms of distribution can be interpreted in terms of variable.

Proposition 1 : We have

$$
\frac{\partial^{j}}{\partial \delta^{j}}[f(y ; 0)]=\sum_{k=1}^{j}(-1)^{k} \frac{\partial^{k}}{\partial y^{k}}\left[a_{j, k}(y) f(y ; 0)\right],
$$


where $a_{j, k}$ is given in 3.5 and 3.3 .

For instance, the first and second-order derivatives of a composite function are :

$$
\begin{aligned}
\frac{d}{d t} g[h(t)] & =g^{(1)}[h(t)] h^{(1)}(t), \\
\frac{d^{2}}{d t^{2}} g[h(t)] & =g^{(2)}[h(t)]\left(h^{(1)}(t)\right)^{2}+g^{(1)}[h(t)] h^{(2)}(t) .
\end{aligned}
$$

Thus we get :

$$
\begin{aligned}
\mathbb{E}\left[g\left(Y_{\delta}\right)\right] & =\mathbb{E}\left[g\left(Y_{0}\right)\right]-\delta \mathbb{E}\left[g^{(1)}\left(Y_{0}\right) \frac{\partial h\left(Y_{0}, \varepsilon, 0\right)}{\partial \delta}\right] \\
& +\frac{\delta^{2}}{2} \mathbb{E}\left[g^{(2)}\left(Y_{0}\right)\left(\frac{\partial h\left(Y_{0}, \varepsilon, 0\right)}{\partial \delta}\right)^{2}+g^{(1)}\left(Y_{0}\right) \frac{\partial^{2} h\left(Y_{0}, \varepsilon, 0\right)}{\partial \delta^{2}}\right]+o\left(\delta^{2}\right) .
\end{aligned}
$$

We deduce the following second-order expansion of the p.d.f :

$$
\begin{aligned}
f(y ; \delta) & =f(y ; 0)-\delta \frac{d}{d y}\left[f(y ; 0) \mathbb{E}\left[\frac{\partial h\left(Y_{0}, \varepsilon, 0\right)}{\partial \delta} \mid Y_{0}=y\right]\right] \\
& +\frac{\delta^{2}}{2}\left\{\frac{d^{2}}{d y^{2}}\left[f(y ; 0) \mathbb{E}\left[\left(\frac{\partial h\left(Y_{0}, \varepsilon, 0\right)}{\partial \delta}\right)^{2} \mid Y_{0}=y\right]\right]-\frac{d}{d y}\left[f(y ; 0) \mathbb{E}\left[\frac{\partial^{2} h\left(Y_{0}, \varepsilon, 0\right)}{\partial \delta^{2}} \mid Y_{0}=y\right]\right]\right\} \\
& +o\left(\delta^{2}\right) .
\end{aligned}
$$

The expansion in terms of variable is greatly simplified when the shock in variable is linear in $\delta$.

Corollary 1 : Let us assume that $Y_{\delta}=Y_{0}+\delta Z\left(Y_{0}, \varepsilon\right)$, say, and denote $\mu_{p}\left(Y_{0}\right)=$ $\mathbb{E}\left[Z^{p}\left(Y_{0}, \varepsilon\right) \mid Y_{0}\right]$ the conditional power moments of the stochastic shock $Z$. We get :

$$
f(y ; \delta)=f(y ; 0)+\sum_{j=1}^{p}\left[\frac{\delta^{j}}{j !}(-1)^{j} \frac{d^{j}}{d y^{j}}\left[f(y ; 0) \mu_{j}(y)\right]\right]+o\left(\delta^{p}\right) .
$$

This specific expansion has been first derived in the literature by Martin, Wilde (2002), Theorem $\mathrm{C}$, based on the analysis of the moment generating function of variable $Y_{\delta}$.

For instance, let us assume that $Z\left(Y_{0}, \varepsilon\right)=a\left(Y_{0}\right) U$, with $U=\Phi^{-1}(\varepsilon)$ and $\Phi$ is the c.d.f. of the standard normal. Then, we get :

$$
\mu_{p}\left(Y_{0}\right)=a^{p}\left(Y_{0}\right) \mathbb{E}\left(U^{p}\right)=a^{p}\left(Y_{0}\right) 2^{-p / 2} \frac{p !}{(p / 2) !}, \text { for } p=2 n, n \in \mathbb{N}-\{0\}, 0, \text { otherwise. }
$$


We deduce that :

$$
f(y ; \delta)=f(y ; 0)+\sum_{j=1}^{p}\left[\frac{\delta^{2 j}}{2^{j}(j !)} \frac{d^{2 j}}{d y^{2 j}}\left[f(y ; 0) a^{2 j}(y)\right]\right]+o\left(\delta^{2 p}\right) .
$$

The expansion of the p.d.f for a shock in terms of variable has been derived above in an indirect way. In special cases, it is possible to get it in a direct way, but the computation is rather cumbersome (see Appendix 3). 


\section{Systematic Shock}

Stress-tests are regularly performed to check the resistance of the financial system. They consist in applying shocks to the balance sheets of the financial institutions (assimilated to risky portfolios). The aim of this section is to discuss the notion of shock by means of a systematic factor.

\subsection{Shocks on tails}

The calibration of the shocks is an important stage in the implementation of stress-test exercises. In practice, the shocks are calibrated to be extreme, that is, they lead to realizations that can deviate significantly from the usual observations of the variables of interest 5 . The simplest form of shock is obtained through a translation of variable of interest, or equivalently thanks to a shift on its distribution (see Figure 1 in the univariate case). For instance, in its 2011 stress-testing exercise on European banks, the European Banking Authority studied an increase of $+1 \%$ on the average default rate of loans, equal to about $+1.5 \%$, in banks' portfolios (see the EBA 2011 Aggregate Report)

With this definition of shock the approaches in terms of variable and distribution look similar. However this definition shows some deficiencies: the shock is deterministic, and concerns the mean of the distribution, not the higher moments generally used to capture risk.

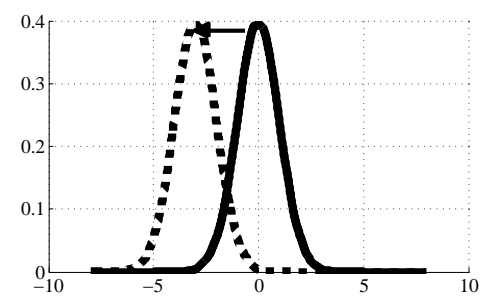

Figure 1: Shift of a distribution. The solid line stands for the baseline univariate distribution.

\footnotetext{
${ }^{5}$ However, as noted above, small shocks may imply large effects in a nonlinear framework. Thus, it is important to consider the effects as a function of $\delta$, that is, the impulse response function [see the discussion in Section 4.2.3].

${ }^{6}$ Such an increase is rather extreme, and should rather be fixed taking into account the quality of the loans in the balance-sheet of each bank. Typically an increase of $+1 \%$ corresponds to a downgrade from $\mathrm{AAA}$ to $\mathrm{BB}$, but only from $\mathrm{BB}$ to $\mathrm{B}$, and can lead to a stable $\mathrm{C}$ rating.
} 
In this Section, we investigate more sophisticated forms of shocks that have been considered in the literature, in terms of either variable, or distribution. We present the link between both approaches in the final part of this Section.

\subsubsection{Shocks on tails in terms of variable}

Richer formulations of the shocks are conceivable. Let us first consider a variable based interpretation, where the shock on $Y_{0}$ is denoted $Y_{\delta}-Y_{0}=h\left(Y_{0}, \varepsilon ; \delta\right)-Y_{0}$. Different forms of shocks on tails are obtained from various dependence structure between the shock and the baseline variable $Y_{0}$. Let us consider a shock such that :

$$
Y_{\delta}=h\left(Y_{0}, \varepsilon ; \delta\right)=Y_{0}+\delta a\left(Y_{0}\right) b(\varepsilon),
$$

where $a$ ( $a$ larger than 0$)$ and $b$ are given functions.

We focus on risks in tails by selecting a function $a$ taking large values in the left and/or right tails of $Y_{0}$. The symmetry or asymmetry of extreme shocks can be managed by an appropriate choice of function $b$. As an illustration, we consider below the following specification :

$$
h\left(Y_{0}, \varepsilon ; \delta\right)=Y_{0}+\delta Y_{0}^{2} \exp \left[-\zeta_{y} Y_{0}\right] \Phi^{-1}(\varepsilon) \exp \left[-\zeta_{\varepsilon} \Phi^{-1}(\varepsilon)\right]
$$

where $\varepsilon$ is an independent variable with Uniform distribution (see Section 2), $\Phi$ is the c.d.f. of the standard Gaussian distribution, and $\zeta_{y}, \zeta_{\varepsilon}$ are two positive scalars.

Four variants are presented in Figure 2: symmetric extreme shock on both tails of $Y_{0}$ $\left(\zeta_{y}=0\right.$ and $\zeta_{\varepsilon}=0$, top left panel in Figure 2), symmetric extreme shock concentrated on the left tail of $Y_{0}\left(\zeta_{y}=0.5\right.$ and $\zeta_{\varepsilon}=0$, bottom left panel), and asymmetric shocks on both tails $\left(\zeta_{y}=0\right.$ and $\zeta_{\varepsilon}=2.5$, top right panel), or on the left tail of $Y_{0}\left(\zeta_{y}=0.5\right.$ and $\zeta_{\varepsilon}=2.5$, bottom right panel). The corresponding unconditional distributions of $Y_{\delta}=h\left(Y_{0}, \varepsilon ; \delta\right)$ are given in Figure 3, which emphasizes the impact of the shock on the thickness of the tails of the distributions, its asymmetry, or its number of modes.

The expansion of the unconditional p.d.f. of $Y_{\delta}$ in 4.2 is derived by Corollary 1. At 

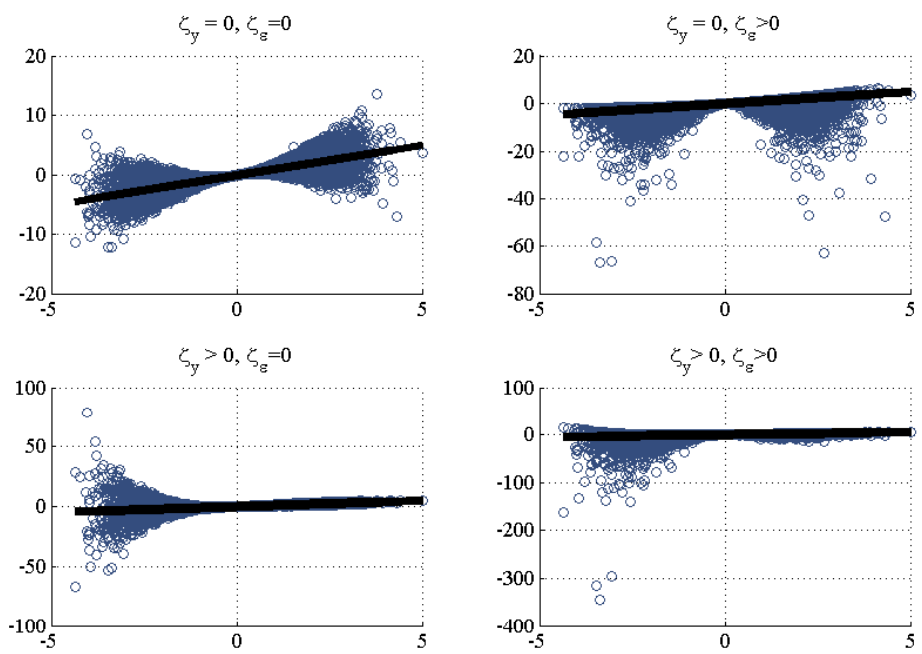

Figure 2: Conditional distributions of the shocked variable $h\left(Y_{0}, \varepsilon ; \delta\right)$ with respect to $Y_{0}$ for different $a$ and $b$ functions in 4.2 . The solid line stands for $h\left(Y_{0}, \varepsilon ; 0\right)=Y_{0}$, and $\Phi^{-1}(\varepsilon)$ has a standard Gaussian distribution.
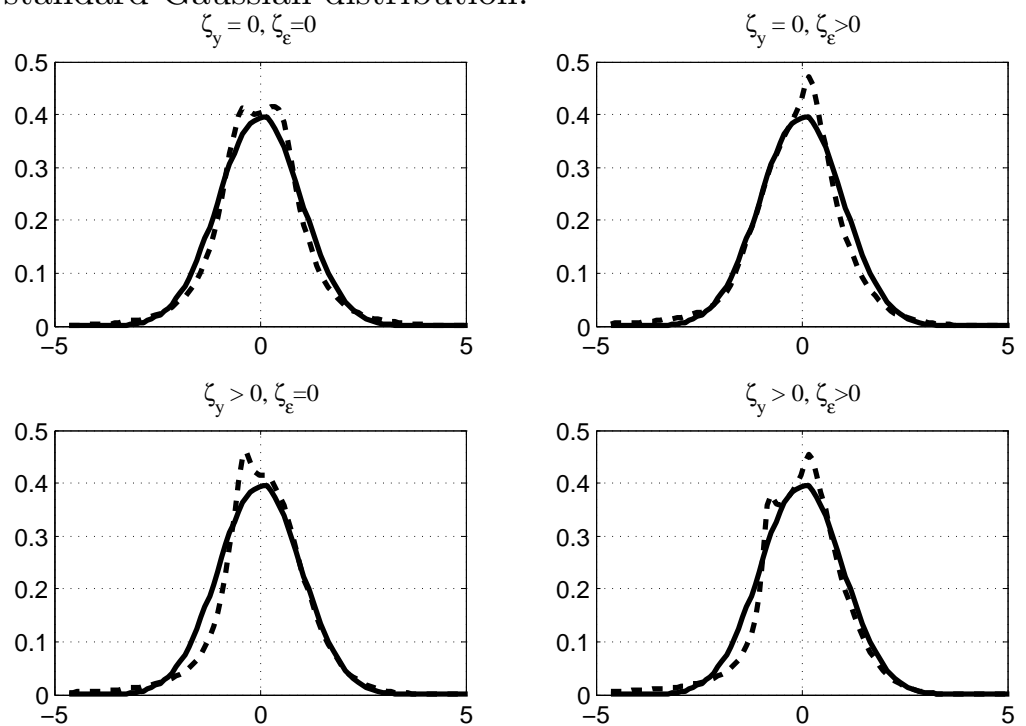

Figure 3: Unconditional distributions of the shocked variable $h\left(Y_{0}, \varepsilon ; \delta\right)$ for different $a$ and $b$ functions in $(4.2)$, where $Y_{0}$ and $\Phi^{-1}(\varepsilon)$ have standard independent Gaussian distributions.

second-order in $\delta$, we get :

$$
\begin{aligned}
f(y ; \delta) & =\phi(y)+\delta \zeta_{\varepsilon} \exp \left[\zeta_{\varepsilon}^{2}\right]\left(2 y-y^{3}-\zeta_{y} y\right) \exp \left[-\zeta_{y} y\right] \phi(y) \\
& +\frac{\delta^{2}}{2}\left(1+4 \zeta_{\varepsilon}^{2}\right) \exp \left[2 \zeta_{\varepsilon}^{2}\right]\left(2 y^{3}-\frac{y^{5}}{2}-\zeta_{y} y^{4}\right) \exp \left[-2 \zeta_{y} y\right] \phi(y),
\end{aligned}
$$


under the assumption of a standard normal variable $Y_{0}$, where $\phi(y)$ is the p.d.f. of the standard normal distribution. The first and second-order moments of $U \exp \left[-\zeta_{\varepsilon} U\right]$, where $U=\Phi^{-1}(\varepsilon)$, are derived by considering the first and second-order derivatives of the Laplace transform of the standard Gaussian variable.

\subsubsection{Shocks on tails in terms of distribution}

Such shocks have been introduced in the literature on robust statistics, which studies the contamination of a baseline distribution by "outliers".

\section{i) Contamination}

A standard specification, introduced in Huber (1964), presents the contaminated distribution as a mixture of a baseline c.d.f. $F(y ; 0)$ and a contaminating c.d.f. $\Xi(y)$ :

$$
F(y ; \delta)=(1-\delta) F(y ; 0)+\delta \Xi(y), \text { with } 0 \leq \delta \leq 1 .
$$

This specification is a special case of shocks in terms of distribution, for which the firstorder expansion in $\delta$ is exact : $f(y ; \delta)=f(y ; 0)+\delta[\xi(y)-f(y ; 0)]$, where $\xi(y)$ is the p.d.f. of the contaminating distribution. A left tail contamination is illustrated in Figure 4, with Gaussian distributions, with different means and the same variance for $F(\bullet ; 0)$ and $\Xi(\bullet)$.

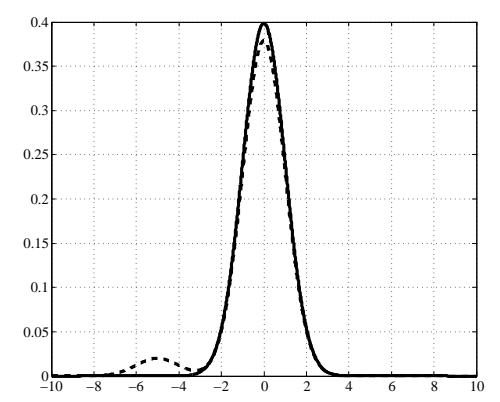

Figure 4: Left tail contaminated distribution. The solid line stands for the baseline distribution, the dashed line for the contaminated distribution.

\section{ii) Contamination in terms of variable}


The conversion of contaminated distribution in terms of variable relies on the general link between families of distributions and families of variables presented in Section 2. Let us for instance apply the Gaussian copula of Section 2.3 to the specification (4.3), where the baseline and contaminated variables are denoted $Y_{0}$ and $Y_{\delta}$, respectively. We have (see Appendix 4 ii)) :

$$
\Phi\left(\sqrt{1-\rho^{2}(\delta)} U+F_{0}\left(Y_{0}\right)\right)=(1-\delta) F_{0}\left(Y_{\delta}\right)+\delta \Xi\left(Y_{\delta}\right)
$$

where $U$ is standard normal and $\rho(0)=1$ to ensure the coherency condition, that is, $Y_{\delta}=Y_{0}$, when $\delta=0$.

\subsubsection{How to reconcile shocks on tails in terms of variable and distribution}

Let us focus on the previous example of contaminated distribution. Several forms of correlation parameter $\rho(\delta)$, and thus several Gaussian copulas, are consistent with contaminated distribution 4.3 , once they satisfy the coherency condition $\rho(0)=1$. This example stresses the difficulty in identifying a unique variable-based specification of a shock on tails expressed in terms of distribution.

Besides, the linearity in $\delta$ of shocks in terms of variable as considered in Section 5.1.1 do not necessarily imply the linearity in $\delta$ of the shocks in terms of distributions as in 4.3. Indeed, let us consider the following approximation of the correlation parameter :

$$
\rho(\delta)=1-\delta^{2} r, \text { where } r=-\left.\frac{\partial \rho(\delta)}{\partial \delta}\right|_{\delta=0},
$$

where the first term in the expansion of $\rho$ is of order $\delta^{2}$ to ensure the constraint $\rho(\delta) \leq 1$, $\forall \delta$. We prove in Appendix 4 iii) that :

$$
Y_{\delta}=Y_{0}+\delta Z+o(\delta), \text { say }
$$

where the variable $Z$ is given by :

$$
Z \approx \frac{\sqrt{2 r} \phi\left(\Phi^{-1}\left(F\left(Y_{0} ; 0\right)\right)\right) U+F\left(Y_{0} ; 0\right)-\Xi\left(Y_{0}\right)}{f\left(Y_{0} ; 0\right)} .
$$

The expansion (4.5)-(4.6) highlights the double effect of the contamination of a distribution in terms of variable: i) a drift effect $\frac{F\left(Y_{0} ; 0\right)-\Xi\left(Y_{0}\right)}{f\left(Y_{0} ; 0\right)}$, and ii) a conditionally heteroscedatic effect $\frac{\sqrt{2 r} \phi\left(\Phi^{-1}\left(F\left(Y_{0} ; 0\right)\right)\right) U}{f\left(Y_{0} ; 0\right)}$ similar to the effects in 4.1), that will impact the tails of the distribution of the contaminated variable. This heteroscedastic effect depends on the 
curvature of function $\rho(\bullet)$ in the neighborhood of $\delta=0$, while the drift effect is negative whenever the contaminating distribution $\Xi$ stochastically dominates at order 1 the baseline distribution $F\left(Y_{0} ; 0\right)$. We still have the multiplicity of interpretations of the shock in terms of variable, since the curvature effect $r$ is unconstrained. In terms of variable, larger is this curvature, larger is the weight on the volatility with respect to the drift.

The comparison of (4.6) with the linear interpretation of the shock in the variable-based approach in 4.1), for which drift effects are missing, emphasizes the differences in both specifications, in spite of their common linear in $\delta$ formulation.

\subsection{Shocks on a systematic factor}

For a vast majority of asset classes, statistical analyses of the price dynamics reveal a limited number of linear, or nonlinear, latent factors, which explain most of the variation in asset prices, or asset returns. In a linear dynamic framework, it is known for instance that one factor drives most of the returns on US Treasury bonds [see e.g. Cochrane, Piazzesi (2005)], while the literature identifies few common linear factors for equity returns [see e.g. Fama, French (1992)].

These systematic factors represent the common dynamic patterns among multiple asset prices, and characterize the dependence structure between assets. For regulators, the identification of linear or nonlinear systematic factors is of crucial importance, since their variations have the biggest impact on institutions' portfolios, as opposed to idiosyncratic factors, whose risk can be diversified away by the holding of a large number of assets. As an illustration, let us consider the Basel 2 credit risk model. Inspired from the Value-ofthe-Firm model [Merton (1974)], it decomposes in a linear way the $\log$ asset/liability $Y_{j}^{*}$ of a firm $j$ in common factor $X$ and specific (or idiosyncratic) components $\eta_{j}$ :

$$
Y_{j}^{*}=\sqrt{\rho} X+\sqrt{1-\rho} \eta_{j}
$$

with positive asset correlation $\rho$, and deduces the default indicator as :

$$
Y_{j}=\mathbb{1}_{Y_{j}^{*}<0}=\mathbb{1}_{\sqrt{\rho} X<-\sqrt{1-\rho} \eta_{j}} .
$$

In the basic model, this default indicator is directly related to the payoff of a Credit Default Swap (CDS) written on firm $j$. Thus, the probability of very large cumulated losses on the institution's portfolio of CDS highly depends in a nonlinear way on the share of common/systematic factors in the distribution of firm's log asset/liability. 
We present below the local expansion of the distribution of variables of interest with respect to "systematic shocks", that are shocks on the distribution of systematic factors, both in terms of distribution and variable.

\subsubsection{Local analysis of systematic shock in terms of distribution}

Let us decompose the joint distribution of variables of interest $f(y ; \delta)$ into two parts: one corresponding to the marginal distribution of the common factors, and the other one referring to the distribution of the variables of interest, conditional on the common factors. A systematic shock would hit the marginal distribution of the common factor $X$ without modifying the conditional distribution of $Y$ given $X$. Thus we get :

$$
f(y ; \delta)=\int f_{1}(y \mid x) f_{2}(x ; \delta) d x .
$$

A direct application of the results in Section 3.1 gives the following local expansion of the distribution of the variable of interest :

$$
f(y ; \delta)=f(y ; 0)+\sum_{j=1}^{p} \frac{\delta^{j}}{j !} \int f_{1}(y \mid x) \frac{\partial^{j} f_{2}(x ; 0)}{\partial \delta^{j}} d x+o\left(\delta^{p}\right) .
$$

In particular, at second-order, we get :

$$
f(y ; \delta)=f(y ; 0)+\delta \int f_{1}(y \mid x) \frac{\partial f_{2}(x ; 0)}{\partial \delta} d x+\frac{\delta^{2}}{2} \int f_{1}(y \mid x) \frac{\partial^{2} f_{2}(x ; 0)}{\partial \delta^{2}} d x+o\left(\delta^{2}\right) .
$$

\subsubsection{Local analysis of systematic shock in terms of variable}

Let us now consider the (vector of) variables of interest $Y_{\delta}$ as a function of common factors $X_{\delta}$, and independent variables $\eta$ :

$$
Y_{\delta}=b\left(X_{\delta}, \eta\right)
$$

where the common factors are such that:

$$
X_{\delta}=h\left(X_{0}, \varepsilon ; \delta\right)
$$

The model involves two types of basic impulses, that are the variable $\eta$ representing the idiosyncratic component and the variable $\varepsilon$ used to define shock on the systematic factor. Equation (4.9) is compatible with nonlinear effects of both types of components 
and possibly cross-effects of systematic and idiosyncratic components.

From Proposition 1, we get at second-order :

$$
\begin{aligned}
f(y ; \delta)= & f(y ; 0)-\delta \int f_{1}(y \mid x) \frac{\partial}{\partial x}\left[f_{2}(x ; 0) \mathbb{E}\left[\frac{\partial h\left(X_{0}, \varepsilon ; \delta\right)}{\partial \delta} \mid X_{0}=x\right]\right] d x \\
+ & \frac{\delta^{2}}{2} \int f_{1}(y \mid x)\left\{\frac{\partial^{2}}{\partial x^{2}}\left[f_{2}(x ; 0) \mathbb{E}\left[\left(\frac{\partial h\left(X_{0}, \varepsilon, 0\right)}{\partial \delta}\right)^{2} \mid X_{0}=x\right]\right]\right. \\
& \left.-\frac{\partial}{\partial x}\left[f_{2}(x ; 0) \mathbb{E}\left[\frac{\partial^{2} h\left(X_{0}, \varepsilon, 0\right)}{\partial \delta^{2}} \mid X_{0}=x\right]\right]\right\} d x+o\left(\delta^{2}\right) .
\end{aligned}
$$

For instance, let us consider a Value-of-the-Firm model for an homogeneous population of $N$ different firms, where $Y_{\delta}^{*}$ is the vector of firms' log asset/liability :

$$
Y_{\delta}^{*}=\sqrt{\rho} \mathbf{1}^{\prime} X_{\delta}+\sqrt{1-\rho} \eta,
$$

where 1 is a $N \times 1$ vector of ones, and $\eta$ has a standard multivariate normal distribution. Let us assume that the single common factor is such that :

$$
X_{\delta}=X_{0}+\delta a\left(X_{0}\right) U
$$

where $U$ is a random variable independent from $X_{0}$, whose second-order moments exists.

We get at second-order :

$$
\begin{aligned}
f_{2}(x ; \delta) & =f_{2}(x ; 0)-\delta \mathbb{E}(U) \frac{d}{d x}\left[a(x) f_{2}(x ; 0)\right]+\frac{\delta^{2}}{2} \mathbb{E}\left(U^{2}\right) \frac{d}{d x}\left[a^{2}(x) f_{2}(x ; 0)\right]+o\left(\delta^{2}\right), \\
f\left(y^{*} ; \delta\right) & =f\left(y^{*} ; 0\right)-\delta \mathbb{E}(U) \int f_{1}\left(y^{*} \mid x\right)\left(a^{(1)}(x) f_{2}(x ; 0)+a(x) f_{2}^{(1)}(x ; 0)\right) d x \\
& +\frac{\delta^{2}}{2} \mathbb{E}\left(U^{2}\right) \int f_{1}\left(y^{*} \mid x\right)\left(2 a(x) a^{(1)}(x) f_{2}(x ; 0)+a^{2}(x) f_{2}^{(1)}(x ; 0)\right) d x+o\left(\delta^{2}\right) .
\end{aligned}
$$

Then this expansion can be used to deduce the expansion of the distribution of the default indicators.

\subsubsection{Systematic shock with extreme effects}

This section helps to precise the notion of extreme shock, which remains rather vague in the literature, and calls for richer specifications of shock than the "shock-in-mean" usually considered in stress-test exercises. When performing stress-tests, we are interested in 
shocks on a systematic factor with extreme impacts on asset portfolio values.

\section{i) Linear dynamic factor model}

In a linear dynamic framework, large effects on the tail of the distribution of variable of interest $Y$ are obtained by introducing shocks on the tails of the factor distribution. This is why the literature usually focus on factor tails (see e.g. Section 4.1).

\section{ii) Nonlinear dynamic factor model}

The situation is very different in the nonlinear framework encountered for portfolios of derivative assets (see e.g. the example of CDS portfolio), or for portfolios managed in some optimal way, for which the relevance of the chosen shocks depends on the nonlinear link between the distribution of portfolio's value and the distribution of the systematic factor.

In such nonlinear factor model, a rather small shock on a factor value can sometimes have a large impact on the portfolio. As an illustration, let us consider an investor, who allocates her/his wealth among two assets, when the asset prices are almost perfectly correlated. Such a large correlation would incite her/him to lever up her/his wealth by selling the less profitable asset to buy the other one. Let us now consider a shock on the joint asset distribution changing the asset correlation to a negative value close to -1 , whereas keeping the expected returns and volatilities at the same level. Under a Gaussian assumption on returns, the joint Gaussian distribution is modified much more in its central part than in its tails. However, the portfolio allocation crystallized at its previous level will be very sensitive to this change, since now the risks lie in the same direction, and the leverage effect is exactly at the opposite of what has to be done. This is exactly the situation encountered by N. Leeson that implied the failure of the Barings, or in the LTCM default. This example shows that a shock on even a central part of the distribution can have extreme consequences. Therefore, it is important to detect the type of shock on the factor with such huge consequences. For this purpose, it is recommended to define a direction of the shock in terms of either variable, or distribution, and to measure the consequences for different levels of $\delta$, that is, to consider an impulse response function [see e.g. Koop, Pesaran, Potter (1996), and Gourieroux, Jasiak (2005)]. 


\section{Stress-testing the European Sovereign Bond Market}

The aim of this section is to avoid the limitations of the current implementation of stresstests, in which the shocks are assumed deterministic and the portfolio is crystallized. In the application presented below, we stress the portfolio of a financial institution invested in European sovereign bonds. We propose a direction of the shock in terms of distribution and variable and show how the riskiness of investor's portfolio, either crystallized or optimally updated, evolves with the size of the shock on the distribution of excess gains.

\section{$5.1 \quad$ Stress-test}

A stress-test requires the application of the different notions introduced in Sections 2, 3, and 4 along the lines of scheme 1.

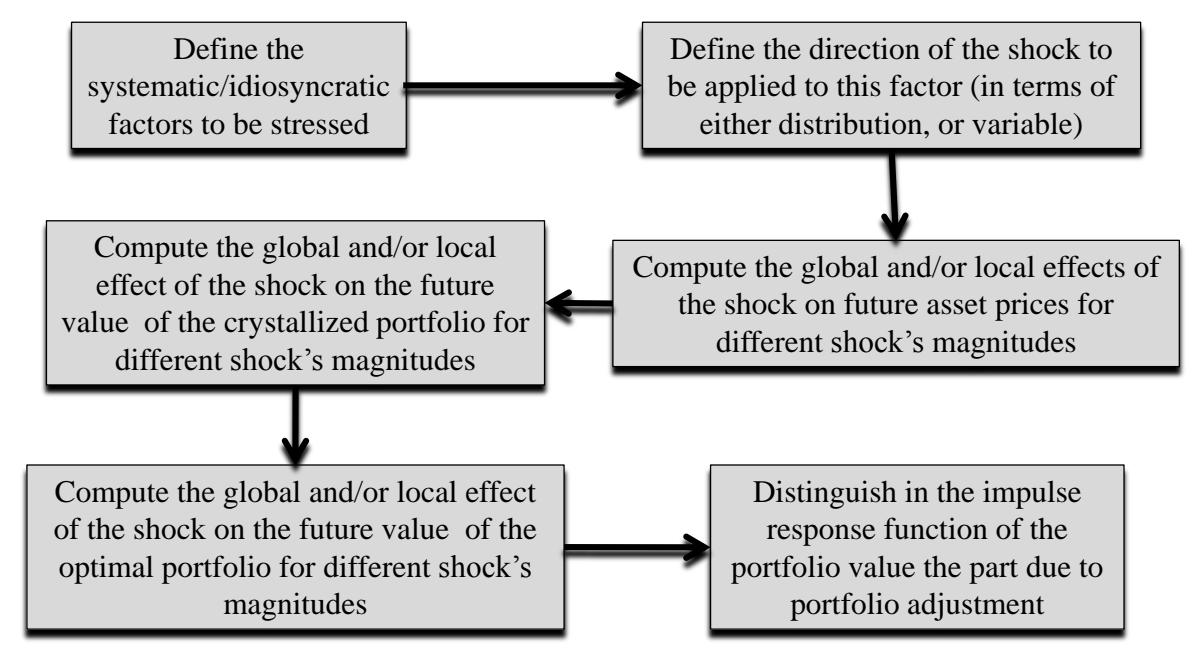

Scheme 1: A stress-test

In the following parts of the section, we apply these different steps to a portfolio of European sovereign bonds. 


\subsection{Excess gains on the European sovereign bond market}

As an illustration, let us consider an investor with mean-variance objective function, who invests her/his wealth in the European sovereign bond market. For simplicity, we consider zero-coupon bonds, with face value 1 , and maturities 10 years. We restrict our sample to six countries representing the variety of the euro area sovereign bond market, that are Germany, France, Italy, Spain, Ireland and Greece. The sample covers the period from July 2001 to June 2011. We assume that the investor has a monthly horizon, and has access to a risk-free asset, which pays the $1 \mathrm{M}$ Eonia swap rate 7 after a one-month holding period. The corresponding monthly excess gains in euro $\$^{8}$ are plotted in Figure 5.

We identify from principal components analysis one systematic factor, which explains
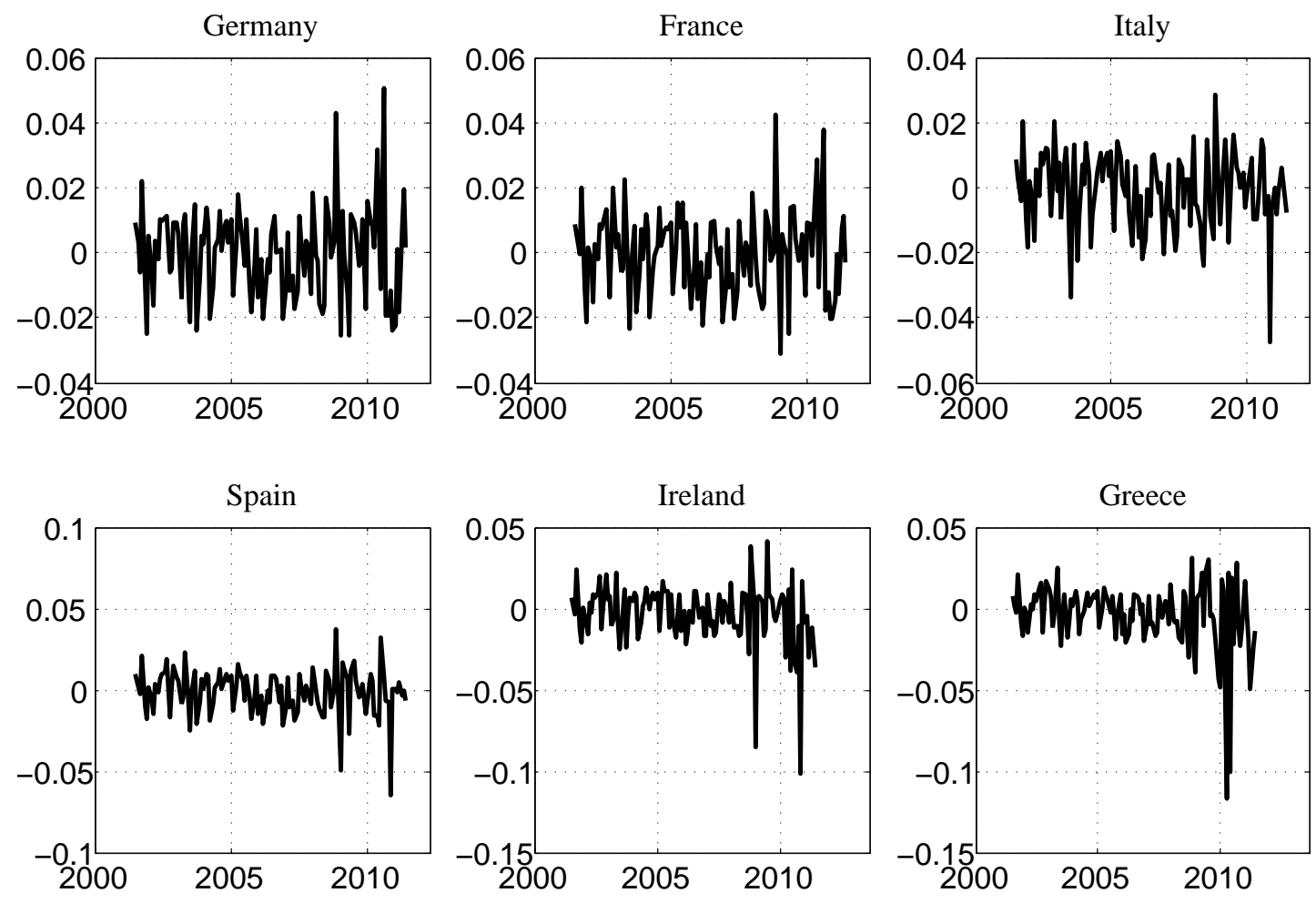

Figure 5: Montly excess gains on 10Y European sovereign bonds.

\footnotetext{
${ }^{7}$ The Eonia swap rate is the fixed rate in a swap contract, whose variable leg is pegged to the Euro OverNight Index Average.

${ }^{8}$ We did not take into account the 1-month maturity effect on bond prices in the computation of excess gains.
} 
about $95 \%$ of the variance of the historical excess gains. The first factor weights uniformly the $10 \mathrm{Y}$ rates of all countries (see Appendix 6). Loosely speaking, this factor is a kind of Eurozone systematic risk. We will shock this "Euro factor" 9 .

As put forward in Figure 6, the first factor has different distributions before and after
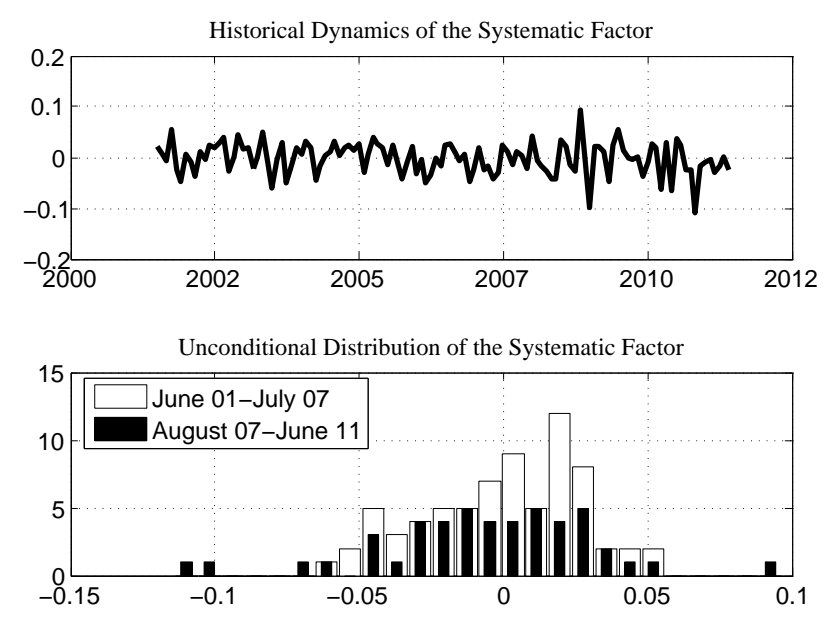

Figure 6: Historical dynamics and histograms of the first systematic factor on periods 2001-2007, and 2007-2011.

July 2007: it features fatter tails, becomes bimodal and seems less asymmetric. We rely on this change to define a systematic shock by contamination.

\subsection{Contamination on the systematic factor}

More precisely, we identify the distribution on the 2001-2007 period of the systematic factor $X$ as our baseline distribution, denoted $F(x ; 0)$, while the distribution on 2007-2011 plays the role of the contaminating distribution $\Xi(x)$.

Then we consider a specification of the shock on the factor's distribution as in Section 4.1.2:

$$
F(x ; \delta)=(1-\delta) F(x ; 0)+\delta \Xi(x), \text { with } 0 \leq \delta \leq 1 .
$$

Let us now consider the contamination in terms of variable. We know that there exists an infinite number of specifications in terms of variable providing the specification (5.1)

\footnotetext{
${ }^{9}$ It would also be possible to consider specific shocks, for instance on the value of the Greek sovereign debt.
} 
of the unconditional distribution of $Y_{\delta}$. Instead of selecting one of these specifications, we consider a linear specification providing locally the equivalence. As shown in Section 4.1.3, equation (4.6), we can express at first-order the empirical contaminated distribution in terms of variable as :

$$
X_{\delta}=X_{0}+\delta Z+o(\delta),
$$

where $X_{0}$ and $X_{\delta}$ are the baseline and contaminated factors, with

$$
Z \approx \frac{\sqrt{2 r} \phi\left(\Phi^{-1}\left(F\left(X_{0} ; 0\right)\right)\right) U+F\left(X_{0} ; 0\right)-\Xi\left(X_{0}\right)}{f\left(X_{0} ; 0\right)},
$$

$U$ is standard Gaussian, and the curvature parameter $r$ is set to $r=2$.

We take advantage of representation (5.3) to simulate a set of 1000 contaminated variables, by drawing independently 1000 times $X_{0}$ in the set of realized $X_{0}$ and $U$ in the standard normal distribution, and by taking $f(\bullet ; 0), F(\bullet ; 0)$, and $\Xi(\bullet)$ at their empirical counterparts $\hat{f}(\bullet ; 0), \hat{F}(\bullet ; 0)$, and $\hat{\Xi}(\bullet)$. The corresponding c.d.f. of the simulated contaminated variables are plotted in Figure 7 for different $\delta$ values. Even if the contamination models in distribution and variable are equivalent in a neighborhood of $\delta=0$, the comparison of Figures 7 and 16 shows that the two specifications imply different types of stochastic shocks for larger values of $\delta$. In particular, the effect on tails is more important with the contamination approach 5.2 written in terms of variable.

\subsection{Impact of the systematic shock on portfolio characteristics}

\section{i) Simulation of contaminated excess-gains}

Let us consider a simple factor model for the vector of excess gains :

$$
P_{\delta}=\mu+\beta^{\prime} X_{\delta}+\Sigma^{1 / 2} \eta
$$

where $\Sigma$ is diagonal, $\eta$ is a standard zero-mean vector, and $\beta$ collects the systematic factor loadings for each bond's excess gain.

The parameters $\mu, \beta, \Sigma$ are estimated from the Seemingly Unrelated Regression (SUR) of excess gains $P_{t}$ on the first factor deduced from the principal component analysis. Then the distribution of $\eta$ is approximated by the historical distribution of the residuals :

$$
\hat{\eta}_{t}=\hat{\Sigma}^{-1 / 2}\left(P_{t}-\hat{\mu}-\hat{\beta}^{\prime} \hat{X}_{t}\right)
$$



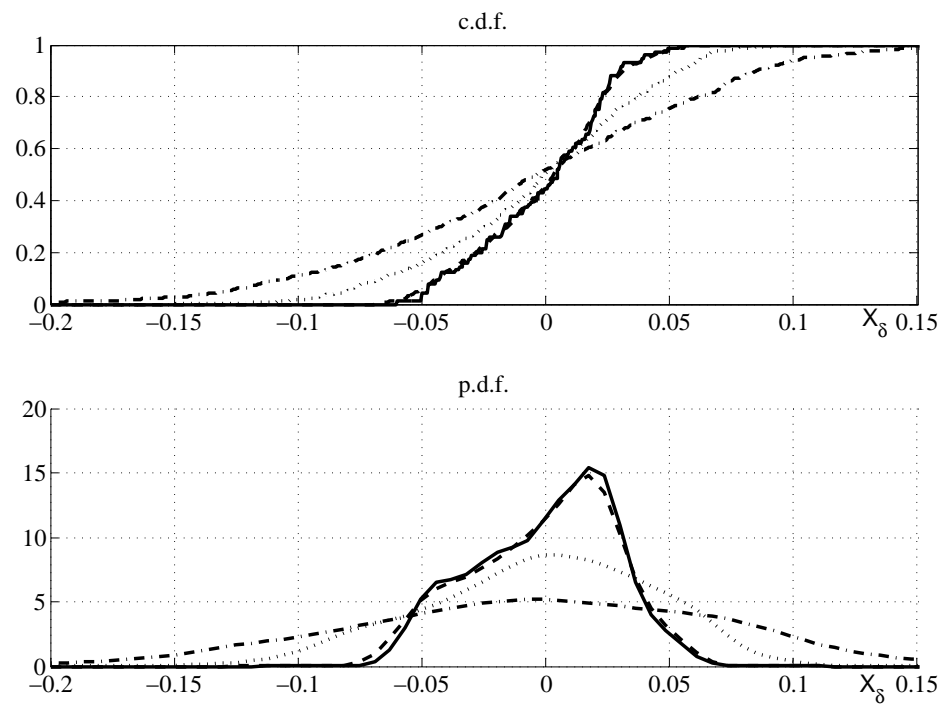

Figure 7: Empirical c.d.f. and p.d.f. of 1000 simulated contaminated factor values $X_{\delta}$ in terms of variable for various $\delta$. The solid line stands for the c.d.f. of the baseline factor $X_{0}$, while dashed, dotted, and dash-dotted lines represent the contaminated empirical distribution for $\delta=0.1,0.5,1$.

The simulation of contaminated excess gains is based on model (5.4) after replacement of the parameters by their estimates. Let us consider a contamination in terms of variable (the contamination in terms of distribution is presented with the corresponding results in Appendix 6). We draw independently a value $\eta^{s}$ in the empirical distribution of the residuals $\hat{\eta}_{t}$, and a value $X_{\delta}^{s}$ in terms of variable as described in Section 5.3. Then the simulated contaminated excess gain is :

$$
P_{\delta}^{s}=\hat{\mu}+\hat{\beta}^{\prime} X_{\delta}^{s}+\hat{\Sigma}^{1 / 2} \eta^{s} .
$$

This procedure is repeated $S=1000$ times. The empirical distribution of the simulated contaminated variables $P_{\delta}^{s}, s=1, \ldots, S$, provides an estimate of the theoretical distribution of the contaminated excess-gains.

\section{ii) The contaminated mean-variance allocation}

We can now derive the mean-variance allocation for an investor, who adjusts herself/himself to the contaminated excess gain distribution. From the estimated distribution of the con- 
taminated excess-gains derived in $5.4 \mathrm{i}$ ), we deduce for each magnitude $\delta$ of the shock the mean and variance/covariance matrix of contaminated excess gains, from which we deduce the optimal mean-variance allocation, denoted $\alpha^{*}(\delta) \sqrt{10}$. The optimal portfolio allocation as a function of the magnitude of the shock $\delta$ is given in Figure 8. This figure highlights the nonlinear effects of $\delta$ on the optimal allocation.

This nonlinearity is a direct consequence of the specification of the stochastic shock and of the mean-variance portfolio management. Indeed, we deduce from (5.2)-(5.4) that:

$$
\begin{aligned}
& \mathbb{E}\left(P_{\delta}\right)=\mu+\beta^{\prime} \mathbb{E}\left(X_{\delta}\right)=\mu+\beta^{\prime} \mathbb{E}\left(X_{0}\right)+\beta^{\prime} \mathbb{E}(Z), \\
& \mathbb{V}\left(P_{\delta}\right)=\beta^{\prime} \mathbb{V}\left(X_{\delta}\right) \beta+\Sigma=\beta^{\prime}\left[\mathbb{V}\left(X_{0}\right)+\delta \operatorname{Cov}\left(X_{0}, Z\right)+\delta \operatorname{Cov}\left(Z, X_{0}\right)+\delta^{2} \mathbb{V}(Z)\right] \beta+\Sigma .
\end{aligned}
$$

Therefore the mean-variance allocation: $\alpha^{*}(\delta)=\gamma^{-1} \mathbb{V}\left(P_{\delta}\right)^{-1} \mathbb{E}\left(P_{\delta}\right)$, has components, which are ratios of polynomials in $\delta$ of degree 12 .

Despite this nonlinear pattern, we observe that these optimal portfolios are short in German, French, Italian and Greek bonds [resp. long in Irish, Spanish bonds] for any value of $\delta$.

\section{iii) Impulse responses for crystallized and optimally adjusted portfolios}

Let us finally compare the properties of the portfolio value under the systematic shock. We consider two portfolio managements, that are i) the mean-variance portfolio crystallized at its optimal level before contamination, and ii) the mean-variance portfolio adjusted for contamination 11 . The properties of the risky part of these portfolios are represented in Figures 9-12 by their mean, variance (volatility), Sharpe performance, VaR and expected shortfall (at the 1\%,5\% and 10\% levels for the two last summaries).

Figures 9-12 emphasize the significant impact of the portfolio management on the characteristic of the portfolio. The performance of the optimal portfolio significantly dominates the crystallized portfolio (see the Sharpe ratio of both portfolios in Figure 9). In this example, the risky part of the crystallized portfolio becomes more volatile and features higher VaR and expected shortfall than the optimal portfolio.

\footnotetext{
${ }^{10}$ We set investor's wealth at 100 , and the level of her/his risk aversion, $\gamma=2$.

${ }^{11}$ As usual in such stress-tests, we assume that the portfolio updating, that is the demand updating by the banks, has no effect on the asset price dynamics.
} 

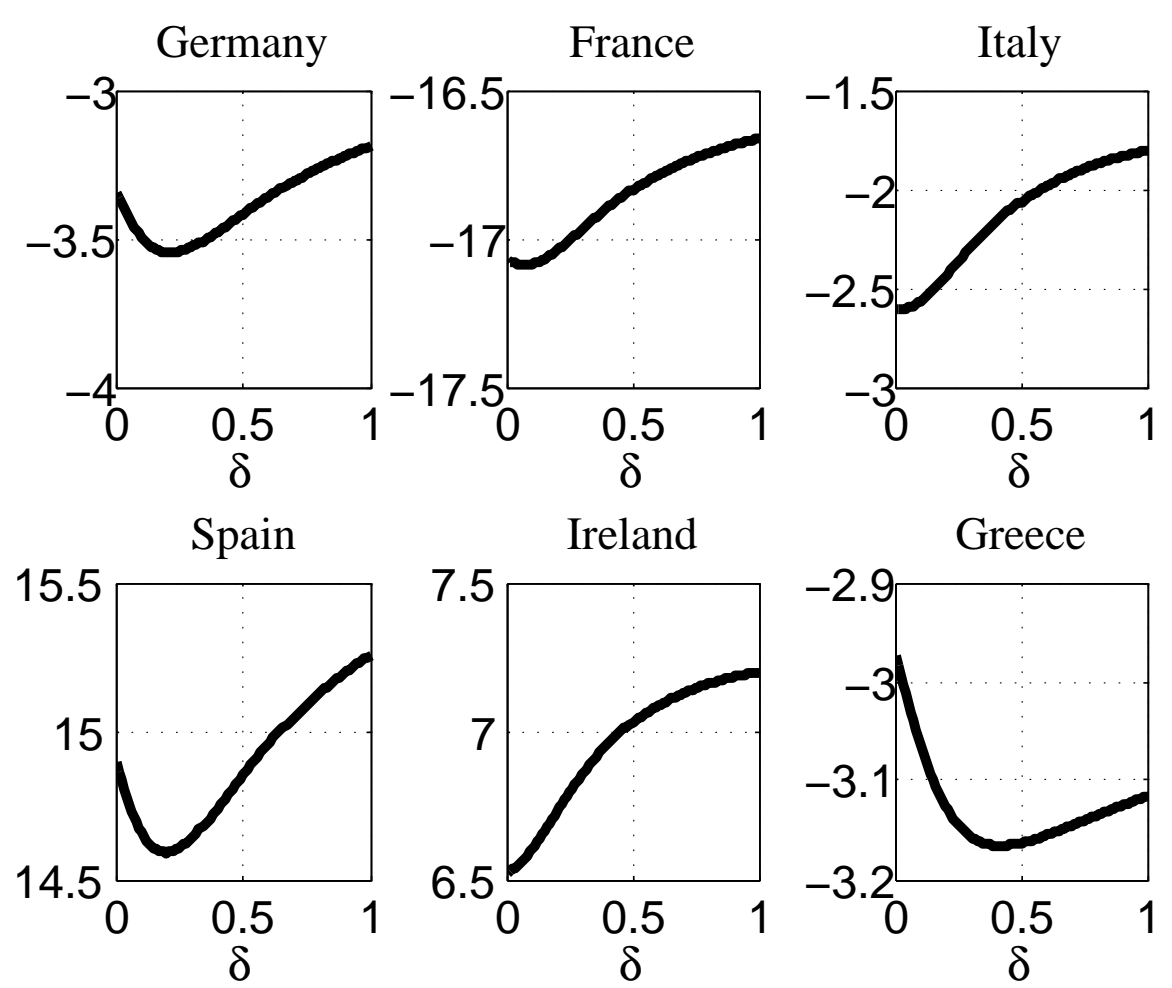

Figure 8: Contaminated mean-variance allocation as a function of $\delta$.
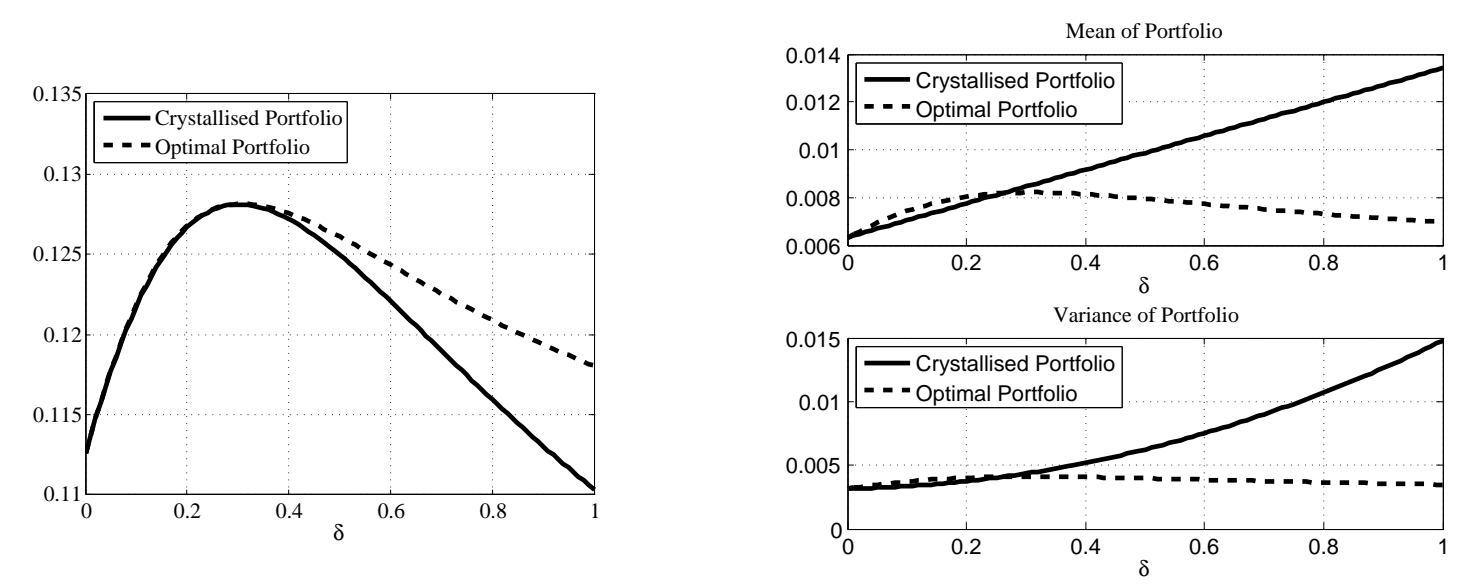

Figure 9: Impulse response of the Sharpe ratio of crystallized and Mean-Variance portfolios (contamination in variable).
Figure 10: Impulse response of the Mean and Variance of crystallized and Mean-Variance portfolios (contamination in variable). 
The local behavior of the VaR for the crystallized and mean-variance portfolios is easily analyzed. The shock in variable is :

$$
P_{\delta}=P_{0}+\delta \beta^{\prime} Z+o(\delta) .
$$

The mean-variance allocation can be expanded as :

$$
\begin{aligned}
\alpha^{*}(\delta) & =\left[\mathbb{V}\left(P_{\delta}\right)\right]^{-1} \mathbb{E}\left(P_{\delta}\right) \\
& =\left[\mathbb{V}\left(P_{0}+\delta \beta^{\prime} Z\right)\right]^{-1} \mathbb{E}\left(P_{0}+\delta \beta^{\prime} Z\right)+o(\delta) \\
& =\left\{\mathbb{V}\left(P_{0}\right)+\delta\left[\operatorname{Cov}\left(P_{0}, \beta^{\prime} Z\right)+\operatorname{Cov}\left(\beta^{\prime} Z, P_{0}\right)\right]\right\}^{-1}\left[\mathbb{E}\left(P_{0}\right)+\delta \mathbb{E}\left(\beta^{\prime} Z\right)\right]+o(\delta) \\
& =\left\{\left[\mathbb{V}\left(P_{0}\right)\right]^{-1}-\delta\left[\mathbb{V}\left(P_{0}\right)\right]^{-1}\left[\operatorname{Cov}\left(P_{0}, \beta^{\prime} Z\right)+\operatorname{Cov}\left(\beta^{\prime} Z, P_{0}\right)\right]\left[\mathbb{V}\left(P_{0}\right)\right]^{-1}\right\}\left[\mathbb{E}\left(P_{0}\right)+\delta \mathbb{E}\left(\beta^{\prime} Z\right)\right]+o(\delta) \\
& =\left[\mathbb{V}\left(P_{0}\right)\right]^{-1} \mathbb{E}\left(P_{0}\right)+\delta\left\{\left[\mathbb{V}\left(P_{0}\right)\right]^{-1} \mathbb{E}\left(\beta^{\prime} Z\right)\right. \\
& \left.-\left[\mathbb{V}\left(P_{0}\right)\right]^{-1}\left[\operatorname{Cov}\left(P_{0}, \beta^{\prime} Z\right)+\operatorname{Cov}\left(\beta^{\prime} Z, P_{0}\right)\right]\left[\mathbb{V}\left(P_{0}\right)\right]^{-1} \mathbb{E}\left(P_{0}\right)\right\}+o(\delta) \\
& =\alpha_{0}+\delta \alpha_{1}, \text { say. }
\end{aligned}
$$

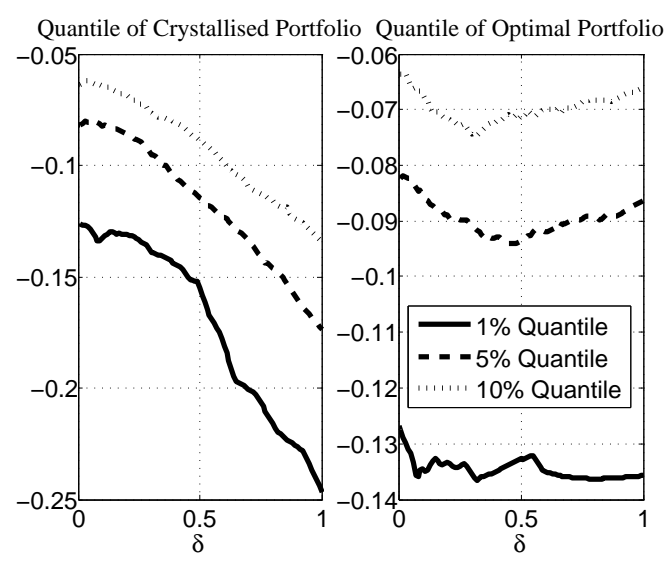

Figure 11: Impulse response of the VaR of crystallized and Mean-Variance portfolios (contamination in variable).
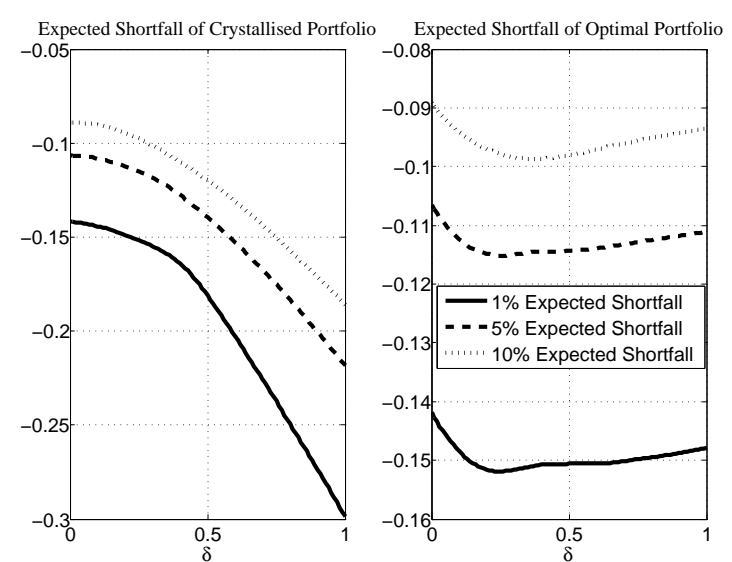

Figure 12: Impulse response of the Expectedshortfall of crystallized and Mean-Variance portfolios (contamination in variable).

The local behavior of the VaR for the crystallized and mean-variance portfolios is easily analyzed. The shock in variable is :

$$
P_{\delta}=P_{0}+\delta \beta^{\prime} Z+o(\delta) .
$$


The mean-variance allocation can be expanded as :

$$
\begin{aligned}
\alpha^{*}(\delta) & =\left[\mathbb{V}\left(P_{\delta}\right)\right]^{-1} \mathbb{E}\left(P_{\delta}\right) \\
& =\left[\mathbb{V}\left(P_{0}+\delta \beta^{\prime} Z\right)\right]^{-1} \mathbb{E}\left(P_{0}+\delta \beta^{\prime} Z\right)+o(\delta) \\
& =\left\{\mathbb{V}\left(P_{0}\right)+\delta\left[\operatorname{Cov}\left(P_{0}, \beta^{\prime} Z\right)+\operatorname{Cov}\left(\beta^{\prime} Z, P_{0}\right)\right]\right\}^{-1}\left[\mathbb{E}\left(P_{0}\right)+\delta \mathbb{E}\left(\beta^{\prime} Z\right)\right]+o(\delta) \\
& =\left\{\left[\mathbb{V}\left(P_{0}\right)\right]^{-1}-\delta\left[\mathbb{V}\left(P_{0}\right)\right]^{-1}\left[\operatorname{Cov}\left(P_{0}, \beta^{\prime} Z\right)+\operatorname{Cov}\left(\beta^{\prime} Z, P_{0}\right)\right]\left[\mathbb{V}\left(P_{0}\right)\right]^{-1}\right\}\left[\mathbb{E}\left(P_{0}\right)+\delta \mathbb{E}\left(\beta^{\prime} Z\right)\right]+o(\delta) \\
& =\left[\mathbb{V}\left(P_{0}\right)\right]^{-1} \mathbb{E}\left(P_{0}\right)+\delta\left\{\left[\mathbb{V}\left(P_{0}\right)\right]^{-1} \mathbb{E}\left(\beta^{\prime} Z\right)\right. \\
& \left.-\left[\mathbb{V}\left(P_{0}\right)\right]^{-1}\left[\operatorname{Cov}\left(P_{0}, \beta^{\prime} Z\right)+\operatorname{Cov}\left(\beta^{\prime} Z, P_{0}\right)\right]\left[\mathbb{V}\left(P_{0}\right)\right]^{-1} \mathbb{E}\left(P_{0}\right)\right\}+o(\delta) \\
& =\alpha_{0}+\delta \alpha_{1}, \text { say. }
\end{aligned}
$$

The value of the mean-variance portfolio is :

$$
Y_{\delta}^{*}=\alpha^{*}(\delta)^{\prime} P_{\delta}=\alpha_{0}^{\prime} P_{0}+\delta\left(\alpha_{0}^{\prime} P_{1}+\alpha_{1}^{\prime} P_{0}\right)+o(\delta),
$$

whereas the value of the crystallized portfolio is equal to :

$$
\tilde{Y}_{\delta}=\alpha_{0}^{\prime}\left(P_{0}+\delta P_{1}\right)+o(\delta) .
$$

We deduce the expansion of the VaR of the mean-variance portfolio :

$$
\begin{aligned}
\operatorname{VaR}_{q}\left(Y_{\delta}^{*}\right) & =\operatorname{VaR}_{q}\left(Y_{0}^{*}\right)+\delta \mathbb{E}\left[\alpha_{0}^{\prime} P_{1}+\alpha_{1}^{\prime} P_{0} \mid Y_{0}^{*}=\operatorname{Va} R_{q}\left(Y_{0}^{*}\right)\right]+o(\delta) \\
& =\operatorname{VaR} q\left(\tilde{Y}_{\delta}\right)+\delta \alpha_{1}^{\prime} \mathbb{E}\left[P_{0} \mid Y_{0}^{*}=\operatorname{VaR}\left(Y_{0}^{*}\right)\right]+o(\delta),
\end{aligned}
$$

by using the expression of the derivative of the VaR [Gourieroux, Laurent, Scaillet (2000)].

Therefore, the difference between the two VaR's is equivalent to :

$$
\begin{aligned}
\frac{1}{\delta}\left[\operatorname{VaR}_{q}\left(Y_{\delta}^{*}\right)-\operatorname{VaR}_{q}\left(\tilde{Y}_{\delta}\right)\right] & \approx \mathbb{E}\left[P_{0} \mid Y_{0}^{*}=\operatorname{VaR}\left(Y_{0}^{*}\right)\right]^{\prime}\left[\mathbb{V}\left(P_{0}\right)\right]^{-1} \beta^{\prime} \mathbb{E}(Z) \\
& -\mathbb{E}\left[P_{0} \mid Y_{0}^{*}=\operatorname{VaR}\left(Y_{0}^{*}\right)\right]^{\prime}\left[\mathbb{V}\left(P_{0}\right)\right]^{-1} \operatorname{Cov}\left(P_{0}, Z\right) \beta\left[\mathbb{V}\left(P_{0}\right)\right]^{-1} \mathbb{E}\left(P_{0}\right) \\
& -\mathbb{E}\left[P_{0} \mid Y_{0}^{*}=\operatorname{VaR}\left(Y_{0}^{*}\right)\right]^{\prime}\left[\mathbb{V}\left(P_{0}\right)\right]^{-1} \beta^{\prime} \operatorname{Cov}\left(Z, P_{0}\right)\left[\mathbb{V}\left(P_{0}\right)\right]^{-1} \mathbb{E}\left(P_{0}\right) .
\end{aligned}
$$

This difference can be of any sign, especially in our framework in which $Z$ and $Y_{0}$ are dependent [see equation (5.3)].

Finally, we present in Figures 13-14 the conditional distribution of the contaminated portfolio value $Y_{\delta}$ with respect to its initial/non-contaminated value $Y_{0}$, which can only be 
derived from a specification of shock in terms of variable. Figures 13-14 emphasize the heteroscedasticity of the shock considered in this exercise: the main effect is concentrated on the central part of the initial distribution. Moreover, the comparison of Figures 13-14 highlights how the optimization of the portfolio allocation circumscribes the shock's impact on the portfolio value, even for large shock's magnitude. This feature is consistent with the low sensitivity to the shock of several risk measures for the optimally adjusted portfolio in this example (see Figures 10-12).

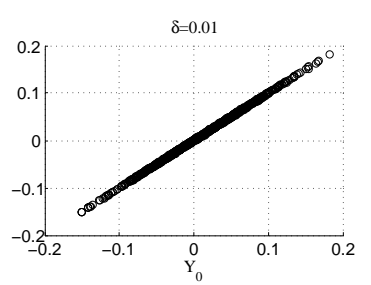

$\delta=0.5$
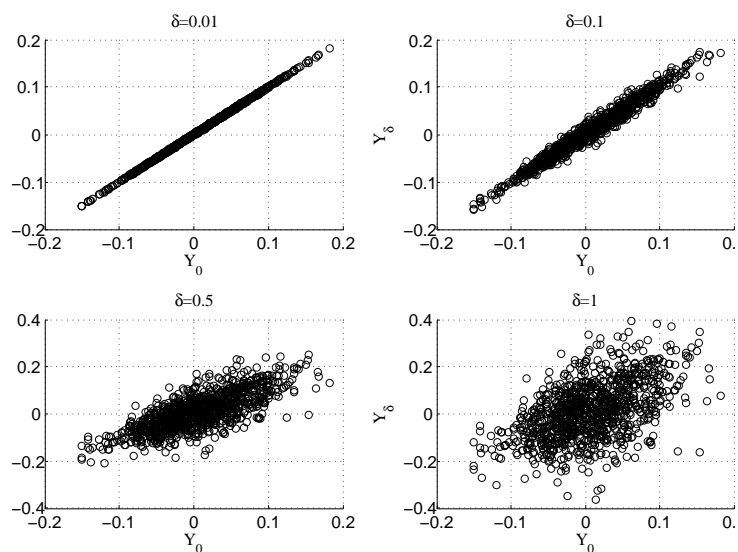

$\delta=1$

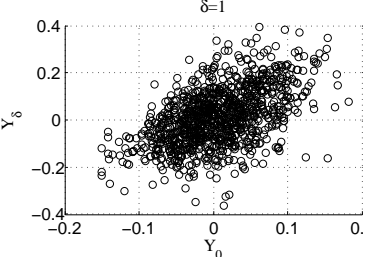

Figure 13: ${ }^{{ }^{0}}$ Impulse response of the conditional distribution of the crystallized portfolio $Y_{\delta} \mid Y_{0}$ (contamination in variable).
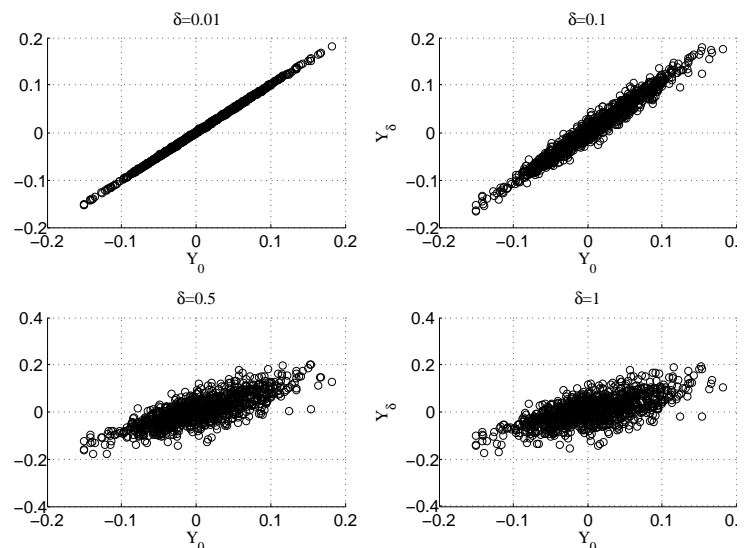

Figure 14: ${ }^{0}$ Impulse response of the conditional distribution of the optimally adjusted portfolio $Y_{\delta} \mid Y_{0}$ (contamination in variable). 


\section{Concluding Remarks}

We have discussed the links between a modeling of shocks in terms of distribution and variable, both for global and local shocks. Such shocks can be introduced on systematic factors to perform stress-tests. This methodology of systematic shock has been illustrated for portfolios of European sovereign bonds. This highlights the different sensitivity to systematic shock of crystallized and optimally updated portfolios.

The main message of our paper is the following : we have seen that a multiplicity of specifications of the shock in terms of variable can lead to a same specification of the shock in terms of distribution. Moreover, the link between these specifications is not obvious: for instance, a linear shock in terms of distribution does not imply a linear shock in terms of variable. Therefore, a prudential approach may consist in considering carefully joint interpretations of a shock, both in terms of distribution and variable. 


\section{R E F E R E N C E S}

Armbruster, B. (2011) : "'A Short Proof of Strassen's Theorem”. Northwestern University Working Paper.

Chesher, A. (1983) : "The Information Matrix Test : Simplified Calculation via a Score Test". Economic Letters, 13, 45-48.

Chesher, A. (1984) : "Testing for Neglected Heterogeneity". Econometrica, 52, 865-872.

Cochrane, J., and M., Piazzesi (2005) : "Bond Risk Premia". American Economic Review, $94,1,138-160$.

European Banking Authority (2011) : "EU-Wide Stress Test Aggregate Report".

Faa di Bruno, F. (1855): "Sullo Sviluppo delle Funzioni". Annali di Suenze Matematiche e Fisische, 6, 479-480.

Fama, E., F., and K., R., French (1992) : "The Cross-Section of Expected Stock Returns". Journal of Finance, 47, 2, 427-465.

Gourieroux, C., and J., Jasiak (2005) : "Nonlinear Impulse Response Function". Annales dÉconomie et de Statistique, 78, 1-33.

Gourieroux, C., Laurent, J-P., and O., Scaillet (2000) : "Sensitivity Analysis of Value-atRisk". Journal of Empirical Finance, 7, 225-245.

Gourieroux, C., and A., Monfort (1995): "Statistic and Econometric Models"'. Vol.2, Cambridge University Press.

Huang, H., Marcantognini, A., and N., Young (2005) : "Chain Rules for Higher Order Derivatives". Working Paper. 
Huber, J., P. (1964) : "Robust Estimation of a Location Parameter". The Annals of Mathematical Statistics, 35, 1, 73-101.

Joe, H. (1997) : "Multivariate Models and Dependence Concepts". Chapman \& Hall, London.

Johnson, W. (2002) : "The Curious History of Faa di Bruno's Formula". American Mathematical Monthly, 109, 217-234.

Koop, G., Pesaran, H., and S., Potter (1996): "'Impulse Response Analysis in Nonlinear Multivariate Models"'. Journal of Econometrics, 74, 119-147.

Martin, R., and T., Wilde (2002) : "Unsystematic Credit Risk", Risk Magazine, 15, 123128.

Merton, R., C. (1974) : "On the Pricing of Corporate Debt : the Risk Structure of Interest Rates". Journal of Finance, 29, 449-470.

Rothschild, M., and J., E. Stiglitz (1970) : "Increasing Risk: 1. A Definition", Journal of Economic Theory, 2, 225-283.

Sklar, A. (1959) : "Fonctions de répartition à N dimensions et leurs marges". Publications de l'Institut de Statistiques de l'Université de Paris, 8, 229-231.

Spindler, K. (2005) : "A Short Proof of the Formula of Faa di Bruno". Elemente der Mathematik, 60, 33-35.

Strassen, V. (1965) : "The Existence of Probability Measure with Given Marginals"'. The Annals of mathematical Statistics, 36, 423-439.

Sydsaeter, K., and P., Hammond (2008) : "Essential Mathematics for Economic Analysis"'. $3^{\text {rd }}$ ed., Prentice Hall. 


\section{Appendix 1 Shocks to Multivariate Distribution}

\section{i) Multivariate Gaussian copula for bidimensional variables}

Definition a.1 : The Gaussian copula for bidimensional variables is the function defined on $[0,1]^{4}$ by :

$$
C\left(u_{1}, u_{2}, v_{1}, v_{2}\right)=\Psi\left[\Phi^{-1}\left(u_{1}\right), \Phi^{-1}\left(u_{2}\right), \Phi^{-1}\left(v_{1}\right), \Phi^{-1}\left(v_{2}\right), R\right],
$$

where $\Psi\left(x_{1}, x_{2}, y_{1}, y_{2}, R\right)$ is the joint c.d.f. of the four-dimensional Gaussian distribution $\mathcal{N}\left(\mathbf{0},\left(\begin{array}{cc}I d_{2} & R \\ R & I d_{2}\end{array}\right)\right)$. The block matrix $R$ is such that $R=\left(\begin{array}{cc}\rho_{11} & \rho_{12} \\ \rho_{21} & \rho_{22}\end{array}\right)$, and $\mathbf{0}$ is a $4 \times 1$ vector of zeros.

The block correlation matrix is constrained by the positive definiteness of the variancecovariance matrix, which imposes that :

$$
\operatorname{det}\left(\begin{array}{ccc}
1 & 0 & \rho_{11} \\
0 & 1 & \rho_{21} \\
\rho_{11} & \rho_{21} & 1
\end{array}\right)=1-\rho_{11}^{2}-\rho_{21}^{2}>0
$$

and

$$
\begin{aligned}
\operatorname{det}\left(\begin{array}{cccc}
1 & 0 & \rho_{11} & \rho_{12} \\
0 & 1 & \rho_{21} & \rho_{22} \\
\rho_{11} & \rho_{21} & 1 & 0 \\
\rho_{12} & \rho_{22} & 0 & 1
\end{array}\right) & =\operatorname{det}(I d) \operatorname{det}\left(I d-R^{\prime} R\right) \\
& =\operatorname{det}\left(I d-R^{\prime} R\right) \\
& =\left(1-\rho_{11}^{2}-\rho_{21}^{2}\right)\left(1-\rho_{12}^{2}-\rho_{22}^{2}\right)-\left(\rho_{11}^{2} \rho_{12}^{2}+\rho_{21}^{2} \rho_{22}^{2}\right)^{2}>0
\end{aligned}
$$

by the block description of the determinant [see e.g. Gourieroux, Monfort (1995), property A.5].

This copula defines a joint c.d.f. for the bivariate vectors: $U=\left(\begin{array}{lll}U_{1} & , U_{2}\end{array}\right)^{\prime}$ and $V=\left(\begin{array}{lll}V_{1} & , V_{2}\end{array}\right)^{\prime}$, which have the same marginal uniform distribution on $[0,1]^{2}$. 


\section{ii) Derivation of the recursive form}

We have now to derive the conditional c.d.f. of $V=\left(\begin{array}{c}V_{1} \\ V_{2}\end{array}\right)$ given $U=\left(\begin{array}{c}U_{1} \\ U_{2}\end{array}\right)$ corresponding to this copula, in order to get interpretations in terms of variables. For this purpose, let us consider two standard Gaussian bivariate variables $Y_{0}$ and $Y_{1}$ :

$$
Y_{0}=\left(\begin{array}{c}
Y_{01} \\
Y_{02}
\end{array}\right), \quad Y_{1}=\left(\begin{array}{c}
Y_{11} \\
Y_{12}
\end{array}\right)
$$

such that $\operatorname{Cov}\left(Y_{0}, Y_{1}\right)=R$.

Then the variables $\varepsilon_{1}=F\left(Y_{11} \mid Y_{0}\right)$ and $\varepsilon_{2}=F\left(Y_{12} \mid Y_{0}, Y_{11}\right)$ follow uniform distributions on $[0,1]$, are independent of each other, and independent of $Y_{0}$. By computing directly the conditional c.d.f., we get:

$$
\begin{aligned}
\varepsilon_{1} & =\Phi\left(\frac{Y_{11}-\mathbb{E}\left(Y_{11} \mid Y_{0}\right)}{\mathbb{V}^{1 / 2}\left(Y_{11} \mid Y_{0}\right)}\right) \\
\varepsilon_{2} & =\Phi\left(\frac{Y_{12}-\mathbb{E}\left(Y_{12} \mid Y_{0}, Y_{11}\right)}{\mathbb{V}^{1 / 2}\left(Y_{12} \mid Y_{0}, Y_{11}\right)}\right) .
\end{aligned}
$$

The conditional means and variances can be computed explicitly. We get:

$$
\begin{aligned}
& \mathbb{E}\left(Y_{11} \mid Y_{0}\right)=\rho_{11} Y_{01}+\rho_{12} Y_{02} \equiv a_{1} Y_{01}+a_{2} Y_{02}, \\
& \mathbb{V}\left(Y_{11} \mid Y_{0}\right)=1-\rho_{11}^{2}-\rho_{12}^{2} \equiv \sigma_{1}^{2}, \\
& \mathbb{E}\left(Y_{12} \mid Y_{0}, Y_{11}\right)=\operatorname{Cov}\left[Y_{12},\left(\begin{array}{c}
Y_{0} \\
Y_{11}
\end{array}\right)\right]\left[\mathbb{V}\left(\begin{array}{c}
Y_{0} \\
Y_{11}
\end{array}\right)\right]^{-1}\left(\begin{array}{c}
Y_{0} \\
Y_{11}
\end{array}\right) \\
& =\left(\begin{array}{ll}
\rho_{12} & \rho_{22}
\end{array}\right)\left[I d-\left(\begin{array}{c}
\rho_{11} \\
\rho_{21}
\end{array}\right)\left(\begin{array}{ll}
\rho_{11} & \rho_{21}
\end{array}\right)\right]^{-1}\left(\begin{array}{c}
Y_{0} \\
Y_{11}
\end{array}\right) \\
& \equiv b_{1} Y_{01}+b_{2} Y_{02}++b_{3} Y_{11} \text {, }
\end{aligned}
$$

by applying the block inversion formula [see e.g. Gourieroux, Monfort (1995) Property A.4]. Moreover :

$$
\mathbb{V}\left(Y_{12} \mid Y_{0}, Y_{11}\right)=1-\left(\begin{array}{ll}
\rho_{12} & \rho_{22}
\end{array}\right)\left[I d-\left(\begin{array}{c}
\rho_{11} \\
\rho_{21}
\end{array}\right)\left(\begin{array}{ll}
\rho_{11} & \rho_{21}
\end{array}\right)\right]^{-1}\left(\begin{array}{c}
\rho_{12} \\
\rho_{22}
\end{array}\right) \equiv \sigma_{2}^{2},
$$


by applying the block inversion formula.

We deduce that:

$$
\left\{\begin{array}{l}
Y_{11}=a_{1} Y_{01}+a_{2} Y_{02}+\sigma_{1} \Phi^{-1}\left(\varepsilon_{1}\right) \\
Y_{12}=b_{1} Y_{01}+b_{2} Y_{02}+b_{3} Y_{11}+\sigma_{2} \Phi^{-1}\left(\varepsilon_{2}\right)
\end{array}\right.
$$

or equivalently the conditional distribution of $V$ given $U$ is such that we get the recursive system of equations:

$$
\left\{\begin{array}{l}
\Phi^{-1}\left(V_{1}\right)=a_{1} \Phi^{-1}\left(U_{1}\right)+a_{2} \Phi^{-1}\left(U_{2}\right)+\sigma_{1} \Phi^{-1}\left(\varepsilon_{1}\right) \\
\Phi^{-1}\left(V_{2}\right)=b_{1} \Phi^{-1}\left(U_{1}\right)+b_{2} \Phi^{-1}\left(U_{2}\right)+b_{3} \Phi^{-1}\left(V_{1}\right)+\sigma_{2} \Phi^{-1}\left(\varepsilon_{2}\right)
\end{array}\right.
$$

where $\varepsilon_{1}, \varepsilon_{2}$ are independent uniform variables independent of $U=\left(\begin{array}{c}U_{1} \\ U_{2}\end{array}\right)$.

Let us finally consider a bivariate vectors $Y_{0}=\left(\begin{array}{c}Y_{01} \\ Y_{02}\end{array}\right), Y_{1}=\left(\begin{array}{c}Y_{11} \\ Y_{12}\end{array}\right)$ with marginal distributions $F_{0}\left(y_{0}\right), F_{1}\left(y_{1}\right)$, say. The marginal distribution of the bivariate vector $Y_{0}$ can be decomposed into the marginal distribution of $Y_{01}$ with c.d.f. $F_{01}\left(y_{01}\right)$ and the conditional distribution of $Y_{02}$ given $Y_{01}$ with conditional c.d.f. $F_{02 \mid 01}\left(y_{02} \mid y_{01}\right)$. Similar notations are introduced for the second bivariate vector, that are $F_{11}\left(y_{11}\right)$ and $F_{12 \mid 11}\left(y_{12} \mid y_{11}\right)$.

The basic uniform variable $U_{1}, U_{2}, V_{1}, V_{2}$ can be chosen such that:

$$
\begin{array}{ll}
U_{1}=F_{01}\left(Y_{01}\right), & U_{2}=F_{02 \mid 01}\left(Y_{02} \mid Y_{01}\right), \\
V_{1}=F_{11}\left(Y_{11}\right), & V_{2}=F_{12 \mid 11}\left(Y_{12} \mid Y_{11}\right) .
\end{array}
$$

Then the two bidimensional stochastic variables $Y_{0}$ and $Y_{1}$ can be linked by a Gaussian copula 6.1.

Proposition a.2 : Let us consider a pair of bidimensional stochastic variables $Y_{0}=$ $\left(\begin{array}{l}Y_{01} \\ Y_{02}\end{array}\right), Y_{1}=\left(\begin{array}{c}Y_{11} \\ Y_{12}\end{array}\right)$, such that the marginal c.d.f of $Y_{01}$ is $F_{01}\left(y_{01}\right)$ and the conditional distribution of $Y_{02}$ given $Y_{01}$ is $F_{02 \mid 01}\left(y_{02} \mid y_{01}\right)$ [resp. $F_{11}\left(y_{11}\right)$ and $\left.F_{12 \mid 11}\left(y_{12} \mid y_{11}\right)\right]$.

Let us denote:

$$
\begin{array}{ll}
U_{1}=F_{01}\left(Y_{01}\right), & U_{2}=F_{02 \mid 01}\left(Y_{02} \mid Y_{01}\right) \\
V_{1}=F_{11}\left(Y_{11}\right), & V_{2}=F_{12 \mid 11}\left(Y_{12} \mid Y_{11}\right)
\end{array}
$$


Vectors $Y_{0}$ and $Y_{1}$ admit a Gaussian copula if and only if the conditional distribution of $Y_{1}$ given $Y_{0}$ can be represented by the system of equations:

$$
\begin{aligned}
& \Phi^{-1}\left[F_{11}\left(Y_{11}\right)\right] \quad=a_{1} \Phi^{-1}\left[F_{01}\left(Y_{01}\right)\right]+a_{2} \Phi^{-1}\left[F_{02 \mid 01}\left(Y_{02} \mid Y_{01}\right)\right]+\sigma_{1} \Phi^{-1}\left(\varepsilon_{1}\right) \\
& \Phi^{-1}\left[F_{12 \mid 11}\left(Y_{12} \mid Y_{11}\right)\right]=b_{1} \Phi^{-1}\left[F_{01}\left(Y_{01}\right)\right]+b_{2} \Phi^{-1}\left[F_{02 \mid 01}\left(Y_{02} \mid Y_{01}\right)\right]+b_{3} \Phi^{-1}\left[F_{11}\left(Y_{11}\right)\right]+\sigma_{2} \Phi^{-1}\left(\varepsilon_{2}\right),
\end{aligned}
$$

where $\varepsilon_{1}, \varepsilon_{2}$ are independent uniform variables, independent of $Y_{0}$, and the coefficients $a_{1}$, $a_{2}, b_{1}, b_{2}, b_{3}, \sigma_{1}, \sigma_{2}$ are function of $R$ given in 6.4 - 6.7).

The extension to parametric families of bivariate variables $Y_{\delta}$ is obtained by making the matrix $R(\delta)$ function of $\delta$. The coherency condition (2.9) then implies $R(0)=I d_{2}$.

\section{ii) Local analysis for multivariate distribution}

Let us consider a multivariate variable $Y_{\delta}=h\left(Y_{0}, \varepsilon ; \delta\right)$, where $Y_{\delta}, Y_{0}, \varepsilon$ are vectors of dimension $N, h\left(Y_{0}, \varepsilon ; \delta\right)=\left(h_{1}(\bullet), \ldots, h_{N}(\bullet)\right)^{\prime}$, and the expectation $\mathbb{E}\left(g\left[Y_{\delta}\right]\right)$, where $g$ is a function of dimension 1. Thus,

$$
\begin{aligned}
\mathbb{E}\left(g\left[Y_{\delta}\right]\right) & =\mathbb{E}\left(g\left[h\left(Y_{0}, \varepsilon ; \delta\right)\right]\right) \\
& =\mathbb{E}\left(g\left(Y_{0}\right)\right)+\delta \mathbb{E}\left(\frac{\partial g\left(Y_{0}\right)}{\partial y^{\prime}} \frac{\partial h\left(Y_{0}, \varepsilon ; 0\right)}{\partial \delta}\right) \\
& +\frac{\delta^{2}}{2} \mathbb{E}\left(\frac{\partial h^{\prime}\left(Y_{0}, \varepsilon ; 0\right)}{\partial \delta} \frac{\partial^{2} g\left(Y_{0}\right)}{\partial y \partial y^{\prime}} \frac{\partial h^{\prime}\left(Y_{0}, \varepsilon ; 0\right)}{\partial \delta}\right)+\frac{\delta^{2}}{2} \mathbb{E}\left(\frac{\partial g\left(Y_{0}\right)}{\partial y^{\prime}} \frac{\partial^{2} h\left(Y_{0}, \varepsilon ; 0\right)}{\partial \delta^{2}}\right)+o\left(\delta^{2}\right) \\
& =\mathbb{E}\left(g\left(Y_{0}\right)\right)+\delta \mathbb{E}\left(\frac{\partial g\left(Y_{0}\right)}{\partial y^{\prime}} \mathbb{E}\left(\frac{\partial h\left(Y_{0}, \varepsilon ; 0\right)}{\partial \delta} \mid Y_{0}\right)\right) \\
& +\frac{\delta^{2}}{2} \mathbb{E}\left(T r\left[\frac{\partial^{2} g\left(Y_{0}\right)}{\partial y \partial y^{\prime}} \mathbb{E}\left(\frac{\partial h^{\prime}\left(Y_{0}, \varepsilon ; 0\right)}{\partial \delta} \frac{\partial h^{\prime}\left(Y_{0}, \varepsilon ; 0\right)}{\partial \delta} \mid Y_{0}\right)\right]\right) \\
& +\frac{\delta^{2}}{2} \mathbb{E}\left(\frac{\partial g\left(Y_{0}\right)}{\partial y^{\prime}} \mathbb{E}\left(\frac{\partial^{2} h\left(Y_{0}, \varepsilon ; 0\right)}{\partial \delta^{2}} \mid Y_{0}\right)\right)+o\left(\delta^{2}\right)
\end{aligned}
$$

Then, we have :

$$
\begin{aligned}
\mathbb{E}\left(\frac{\partial g\left(Y_{0}\right)}{\partial y^{\prime}} \mathbb{E}\left(\frac{\partial h\left(Y_{0}, \varepsilon ; 0\right)}{\partial \delta} \mid Y_{0}\right)\right) & =\sum_{j=1}^{N} \mathbb{E}\left[\frac{\partial g\left(Y_{0}\right)}{\partial y_{j}} \mathbb{E}\left(\frac{\partial h_{j}\left(Y_{0}, \varepsilon ; 0\right)}{\partial \delta}\right)\right] \\
& =-\sum_{j=1}^{N} \mathbb{E}\left[\frac{g\left(Y_{0}\right)}{f\left(Y_{0} ; 0\right)} \frac{d}{d y_{j}}\left[\mathbb{E}\left(\frac{\partial h_{j}\left(Y_{0}, \varepsilon ; 0\right)}{\partial \delta} \mid Y_{0}=y\right) f(y ; 0)\right]\right]
\end{aligned}
$$


Similarly, we have :

$\mathbb{E}\left(\frac{\partial g\left(Y_{0}\right)}{\partial y^{\prime}} \mathbb{E}\left(\frac{\partial^{2} h\left(Y_{0}, \varepsilon ; 0\right)}{\partial \delta^{2}} \mid Y_{0}\right)\right)=-\sum_{j=1}^{N} \mathbb{E}\left(\frac{g\left(Y_{0}\right)}{f\left(Y_{0} ; 0\right)} \frac{d}{d y_{j}}\left[\mathbb{E}\left(\frac{\partial^{2} h_{j}\left(Y_{0}, \varepsilon ; 0\right)}{\partial \delta^{2}} \mid Y_{0}=y\right) f(y ; 0)\right]\right)$,

and

$$
\begin{aligned}
& \mathbb{E}\left(\operatorname{Tr}\left[\frac{\partial^{2} g\left[Y_{0}\right]}{\partial y \partial y^{\prime}} \mathbb{E}\left(\frac{\partial h^{\prime}\left(Y_{0}, \varepsilon ; 0\right)}{\partial \delta} \frac{\partial h^{\prime}\left(Y_{0}, \varepsilon ; 0\right)}{\partial \delta} \mid Y_{0}=y\right)\right]\right) \\
& =\sum_{j=1}^{N} \sum_{k=1}^{N} \frac{d}{d y_{j} d y_{k}} \mathbb{E}\left(\frac{g\left(Y_{0}\right)}{f\left(Y_{0} ; 0\right)}\left[\mathbb{E}\left(\frac{\partial h_{j}\left(Y_{0}, \varepsilon ; 0\right)}{\partial \delta} \frac{\partial h_{k}\left(Y_{0}, \varepsilon ; 0\right)}{\partial \delta} \mid Y_{0}=y\right) f(y ; 0)\right]\right)
\end{aligned}
$$

Thus the multivariate equivalent of Proposition 1 is :

$$
\begin{aligned}
\frac{\partial}{\partial \delta}[f(y ; 0)] & =-\sum_{j=1}^{N} \frac{d}{d y_{j}}\left[\mathbb{E}\left(\frac{\partial h_{j}\left(Y_{0}, \varepsilon ; 0\right)}{\partial \delta} \mid Y_{0}=y\right) f(y ; 0)\right] \\
\frac{\partial^{2}}{\partial \delta^{2}}[f(y ; 0)] & =\sum_{j=1}^{N} \sum_{k=1}^{N} \frac{d}{d y_{j} d y_{k}}\left[\mathbb{E}\left(\frac{\partial h_{j}\left(Y_{0}, \varepsilon ; 0\right)}{\partial \delta} \frac{\partial h_{k}\left(Y_{0}, \varepsilon ; 0\right)}{\partial \delta} \mid Y_{0}=y\right) f(y ; 0)\right] \\
& -\sum_{j=1}^{N} \frac{d}{d y_{j}}\left[\mathbb{E}\left(\frac{\partial^{2} h_{j}\left(Y_{0}, \varepsilon ; 0\right)}{\partial \delta^{2}} \mid Y_{0}=y\right) f(y ; 0)\right] .
\end{aligned}
$$

\section{Appendix 2}

\section{Proof of Lemma 2}

The result is obtained by a sequence of integration by part. Let us denote $(\underline{y}, \bar{y})$ the support of the function $g$. We get :

$$
\begin{aligned}
\mathbb{E}\left[g^{(k)}\left(Y_{0}\right) a\left(Y_{0}\right)\right] & =\int_{\underline{y}}^{\bar{y}} g^{(k)}(y) a(y) f(y ; 0) d y \\
& =\left[g^{(k-1)}(y) a(y) f(y ; 0)\right]_{\underline{y}}^{\bar{y}}-\int_{\underline{y}}^{\bar{y}} g^{(k-1)}(y) \frac{d}{d y}[a(y) f(y ; 0)] d y \\
& \left.=-\int_{\underline{y}}^{\bar{y}} g^{(k-1)}(y) \frac{d}{d y}[a(y) f(y ; 0)] d y \text { (since } g(\underline{y})=g(\bar{y})=0\right) \\
& =(-1)^{k} \int_{\underline{y}}^{\bar{y}} g(y) \frac{d^{k}}{d y^{k}}[a(y) f(y ; 0)] d y \text { (by applying recursively the same argument) } \\
& =(-1)^{k} \mathbb{E}\left[\frac{g\left(Y_{0}\right)}{f\left(Y_{0} ; 0\right)} \frac{d^{k}}{d y^{k}}\left[a\left(Y_{0}\right) f\left(Y_{0} ; 0\right)\right]\right] .
\end{aligned}
$$




\section{Appendix 3}

\section{An alternative derivation of the expansion in Corollary 1}

Let us consider the specification $Y_{\delta}=h\left(Y_{0}, U ; \delta\right)=Y_{0}+\delta a\left(Y_{0}\right) U$, where $U$ is a variable independent of $Y_{0}$ with p.d.f. $g(u), \delta \geq 0, a(\bullet)>0$. We assume that :

Assumption A1 : Given $Y_{0}$ and $\delta$, there is an increasing bijective relationship between $U$ and $Y_{\delta}$;

Assumption A2 : Given $Y_{\delta}$ and $\delta$, there is an increasing bijective relationship between $U$ and $Y_{0}$.

Under Assumption $A_{1}$, we can write :

$$
u=\frac{y-y_{0}}{\delta a\left(y_{0}\right)}
$$

Then the p.d.f. of $Y_{\delta}$ conditional to $Y_{0}$ is :

$$
f\left(y \mid y_{0} ; \delta\right)=\frac{1}{\delta a\left(y_{0}\right)} g\left(\frac{y-y_{0}}{\delta a\left(y_{0}\right)}\right),
$$

and the unconditional p.d.f of $Y_{\delta}$ is :

$$
f(y ; \delta)=\int\left[\delta a\left(y_{0}\right)\right]^{-1} g\left(\frac{y-y_{0}}{\delta a\left(y_{0}\right)}\right) f\left(y_{0} ; 0\right) d y_{0} .
$$

Under Assumption $A_{2}$, we have :

$$
y=y_{0}+\delta a\left(Y_{0}\right) u \Leftrightarrow y_{0}=c(y, u ; \delta), \text { say. }
$$

Let us now consider the change of variable $y_{0} \rightarrow u$ in integral (6.8). We get :

$$
f(y ; \delta)=\int[\delta a(c(y, u ; \delta))]^{-1} g(u) f(c(y, u ; \delta) ; 0)\left|\frac{\partial c(y, u ; \delta)}{\partial u}\right| d u .
$$

At first-order in $\delta$ we get : $c(y, u ; \delta) \approx y-\delta a(y) u$. Thus, 6.9 becomes :

$$
\begin{aligned}
f(y ; \delta) & \approx \int \frac{a(y)}{a(y-\delta a(y) u)} g(u) f(y-\delta a(y) u ; 0) d u \\
& \approx \int\left[1-\delta a^{(1)}(y) u\right]^{-1} g(u)\left[f(y ; 0)-\delta f^{(1)}(y ; 0) a(y) u\right] d u \\
& \approx \int\left[1+\delta a^{(1)}(y) u\right] g(u)\left[f(y ; 0)-\delta f^{(1)}(y ; 0) a(y) u\right] d u \\
& \approx \int g(u) f(y ; 0) d u-\delta \int\left[f^{(1)}(y ; 0) a(y) u-a^{(1)}(y) u f(y ; 0)\right] g(u) d u,(6.10)
\end{aligned}
$$


,which is the first term in expansion of Corollary 1.

\section{Appendix 4}

From a distribution-based to a variable-based approach with Gaussian copula

The Gaussian copula is given by :

$$
C(u, v)=\Psi\left[\Phi^{-1}(u), \Phi^{-1}(v), \rho\right],
$$

where $\Psi(x, y, \rho)$ is the joint c.d.f. of the bivariate Gaussian distribution $\mathcal{N}\left(\left(\begin{array}{l}0 \\ 0\end{array}\right),\left(\begin{array}{ll}1 & \rho \\ \rho & 1\end{array}\right)\right)$.

i) Derivatives of equation (2.4)

Let us consider a Gaussian copula with correlation parameter $\rho$ and two variables $Y_{0}, Y_{1}$, whose marginal distributions are standard normal. We have from 2.4 :

$$
\begin{aligned}
\varepsilon & =F_{1 \mid 0}\left(Y_{1} \mid Y_{0}\right) \\
& =\frac{\partial C}{\partial u}\left[F_{0}\left(Y_{0}\right), F_{1}\left(Y_{1}\right)\right] \\
& =\Phi\left(\frac{\Phi^{-1}(V)-\rho \Phi^{-1}(U)}{\sqrt{1-\rho^{2}}}\right)
\end{aligned}
$$

where :

$$
V=\Phi\left(Y_{1}\right), U=\Phi\left(Y_{0}\right)
$$

Therefore :

$$
\Phi^{-1}(V)=\rho \Phi^{-1}(U)+\sqrt{1-\rho^{2}} \Phi^{-1}(\varepsilon),
$$

where $\varepsilon$ has a uniform distribution on $[0 ; 1]$. The result is easily extended to families of distribution by taking Gaussian copulas with correlation parameter $\rho(\delta)$ indexed by $\delta$.

\section{ii) Contamination}


Let us now consider the contamination of Section 4.1.2 in the Gaussian copula case :

$$
F(y ; \delta)=(1-\delta) F(y ; 0)+\delta \Xi(y)
$$

Thus :

$$
\begin{aligned}
\varepsilon & =\Phi\left(\frac{\Phi^{-1}\left(F\left(Y_{\delta} ; \delta\right)\right)-\rho(\delta) \Phi^{-1}\left(F\left(Y_{0} ; 0\right)\right)}{\sqrt{1-\rho^{2}(\delta)}}\right) \\
& =\Phi\left(\frac{\Phi^{-1}\left((1-\delta) F\left(Y_{\delta} ; 0\right)+\delta \Xi\left(Y_{\delta}\right)\right)-\rho(\delta) \Phi^{-1}\left(F\left(Y_{0} ; 0\right)\right)}{\sqrt{1-\rho^{2}(\delta)}}\right)
\end{aligned}
$$

which gives :

$$
\Phi\left(\sqrt{1-\rho^{2}(\delta)} U+\rho(\delta) \Phi^{-1}\left(F\left(Y_{0} ; 0\right)\right)\right)=(1-\delta) F\left(Y_{\delta} ; 0\right)+\delta \Xi\left(Y_{\delta}\right),
$$

where $U=\Phi^{-1}(\varepsilon)$ is a standard Gaussian variable.

\section{iii) First-order expansion}

Let us now assume that $\rho(\delta)=1-\delta^{2} r+o\left(\delta^{2}\right)$, where $r=-\left.\frac{\partial \rho(\delta)}{\partial \delta}\right|_{\delta=0}$, and approximate $Y_{\delta}$ at first-order :

$$
Y_{\delta}=Y_{0}+\delta Z+o(\delta)
$$

More precisely, let us consider the two sides of equation 6.12 . We get for the left hand side :

$$
\begin{aligned}
\Phi\left(\sqrt{1-\rho^{2}(\delta)} U+\rho(\delta) \Phi^{-1}\left(F\left(Y_{0} ; 0\right)\right)\right) & \approx \Phi\left(\sqrt{2 r \delta^{2}} U+\Phi^{-1}\left(F\left(Y_{0} ; 0\right)\right)\right) \\
& \approx F\left(Y_{0} ; 0\right)+\delta \sqrt{2 r} \phi\left(\Phi^{-1}\left(F\left(Y_{0} ; 0\right)\right) U(, 6.13)\right.
\end{aligned}
$$

and

$$
\begin{aligned}
(1-\delta) F\left(Y_{\delta} ; 0\right)+\delta \Xi\left(Y_{\delta}\right) & =F\left(Y_{\delta} ; 0\right)+\delta\left(\Xi\left(Y_{\delta}\right)-F\left(Y_{\delta} ; 0\right)\right) \\
& \approx F\left(Y_{0} ; 0\right)+\delta Z f\left(Y_{0} ; 0\right)+\delta\left(\Xi\left(Y_{0}\right)-F\left(Y_{0} ; 0\right)\right), \text { for the right hand side. }
\end{aligned}
$$

This provides the expression of variable $Z$, that is 4.6 , by identification. 


\section{Appendix 5}

\section{Application: contamination in terms of variable}

\begin{tabular}{|cccccc|}
\hline GE & FR & IT & SP & IR & GR \\
\hline-0.9668 & -0.8860 & -0.9037 & -0.7851 & -0.9293 & -0.7971 \\
\hline
\end{tabular}

Table 1: Mean of excess gains on 2001-2007. All numbers must be divided by 1000 .

\begin{tabular}{|cccccc|}
\hline GE & FR & IT & SP & IR & GR \\
\hline 0.9759 & 0.3775 & -1.2251 & -2.1459 & -7.3262 & -8.9894 \\
\hline
\end{tabular}

Table 2: Mean of excess gains on 2007-2011. All numbers must be divided by 1000 .

\begin{tabular}{|c|cccccc|}
\hline & GE & FR & IT & SP & IR & GR \\
\hline GE & 0.1253 & 0.1164 & 0.1206 & 0.1195 & 0.1247 & 0.1131 \\
FR & 0.1164 & 0.1252 & 0.1196 & 0.1235 & 0.1292 & 0.1173 \\
IT & 0.1206 & 0.1196 & 0.1278 & 0.1215 & 0.1263 & 0.1153 \\
SP & 0.1195 & 0.1235 & 0.1215 & 0.1274 & 0.1306 & 0.1201 \\
IR & 0.1247 & 0.1292 & 0.1263 & 0.1306 & 0.1386 & 0.1238 \\
GR & 0.1131 & 0.1173 & 0.1153 & 0.1201 & 0.1238 & 0.1166 \\
\hline
\end{tabular}

Table 3: Covariance matrix of excess gains on 2001-2007. All numbers must be divided by 1000 .

\begin{tabular}{|c|cccccc|}
\hline & GE & FR & IT & SP & IR & GR \\
\hline GE & 0.2838 & 0.2346 & 0.1140 & 0.1370 & 0.1491 & 0.0496 \\
FR & 0.2346 & 0.2224 & 0.1253 & 0.1673 & 0.2023 & 0.1023 \\
IT & 0.1140 & 0.1253 & 0.1567 & 0.1838 & 0.2239 & 0.1436 \\
SP & 0.1370 & 0.1673 & 0.1838 & 0.2923 & 0.3545 & 0.2524 \\
IR & 0.1491 & 0.2023 & 0.2239 & 0.3545 & 0.6431 & 0.3645 \\
GR & 0.0496 & 0.1023 & 0.1436 & 0.2524 & 0.3645 & 0.8449 \\
\hline
\end{tabular}

Table 4: Covariance matrix of excess gains on 2007-2011. All numbers must be divided by 1000 . 

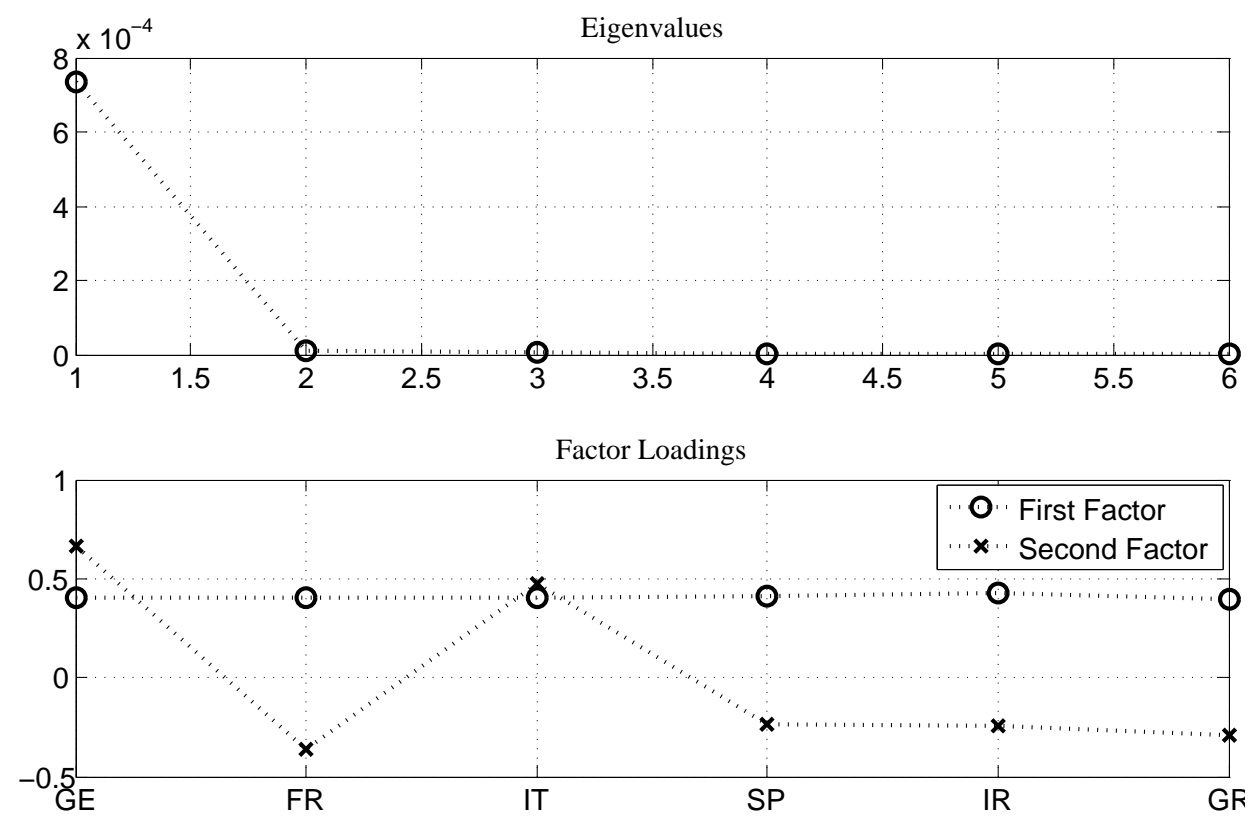

Figure 15: Eigenvalues and factor loadings from the principal components analysis of the 2001-2011 excess gains covariance matrix

\section{Appendix 6}

\section{Application: contamination in terms of distribution}

Let us perform the same application as in Section 6, when the contamination of the systematic factor is expressed in terms of distribution. In this case, we rely on the specification (4.3) to simulate the contaminated factors from a mixture of the baseline distribution of the factor with c.d.f. $F(x ; 0)$, which is estimated on the 2001-2007 sample, and a contaminating distribution with c.d.f. $\Xi(x)$ inferred from the 2007-2011 period :

$$
F(x ; \delta)=(1-\delta) F(x ; 0)+\delta \Xi(x), \text { with } 0 \leq \delta \leq 1 .
$$

More precisely, we proceed in three steps :

1. We draw a set of $S=1000$ uniform independent variables $\left(\omega^{s}\right)_{s=1 \ldots S}$ on $[0,1]$, and two sets of $S=1000$ non-contaminated and contaminated factors $\left[\left(X_{0}^{s}\right)_{s=1 \ldots S}\right.$ and $\left(X_{1}^{s}\right)_{s=1 \ldots S}$, respectively] from the realized factors on 2001-2007, and 2007-2011. 
2. For a given $\delta$ we compute the variable :

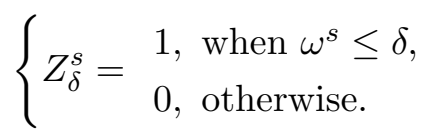

3. Finally, we compute the contaminated factor :

$$
X_{\delta}^{s}=\left(1-Z_{\delta}^{s}\right) X_{0}^{s}+Z_{\delta}^{s} X_{1}^{s}
$$

The empirical distribution of the simulated factor is presented in Figure 16 for $\delta=0,0.1$, 0.5 , and 1 , while the properties of the crystallized and optimally adjusted mean-variance portfolios are plotted in Figures 16-20. As in the contamination in terms of variable, the characteristics of the optimal mean-variance portfolio deviates significantly from the crystallized portfolio's ones. As expected the analysis in terms of variable and distribution look similar for small $\delta$, but they can deviate significantly when $\delta$ becomes larger.
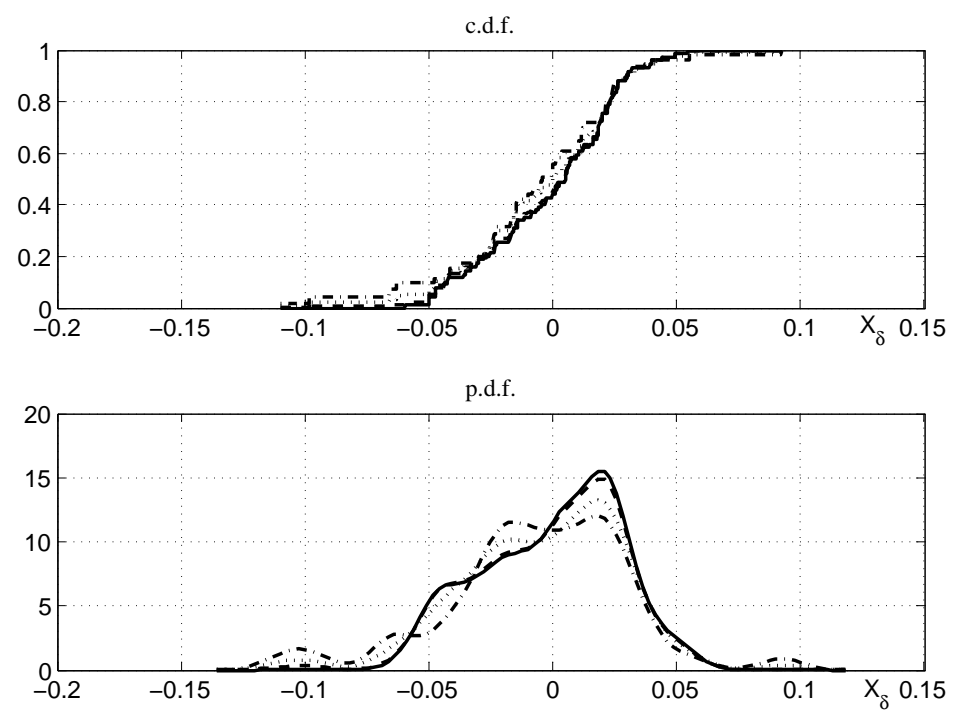

Figure 16: Empirical c.d.f and p.d.f. of 1000 simulated factor $X_{\delta}$, contaminated in terms of distribution for various $\delta$. The solid line stands for the c.d.f. of the baseline factor $X_{0}$, while dashed, dotted, and dash-dotted lines represent the contaminated empirical distribution for $\delta=0.1,0.5,1$. 

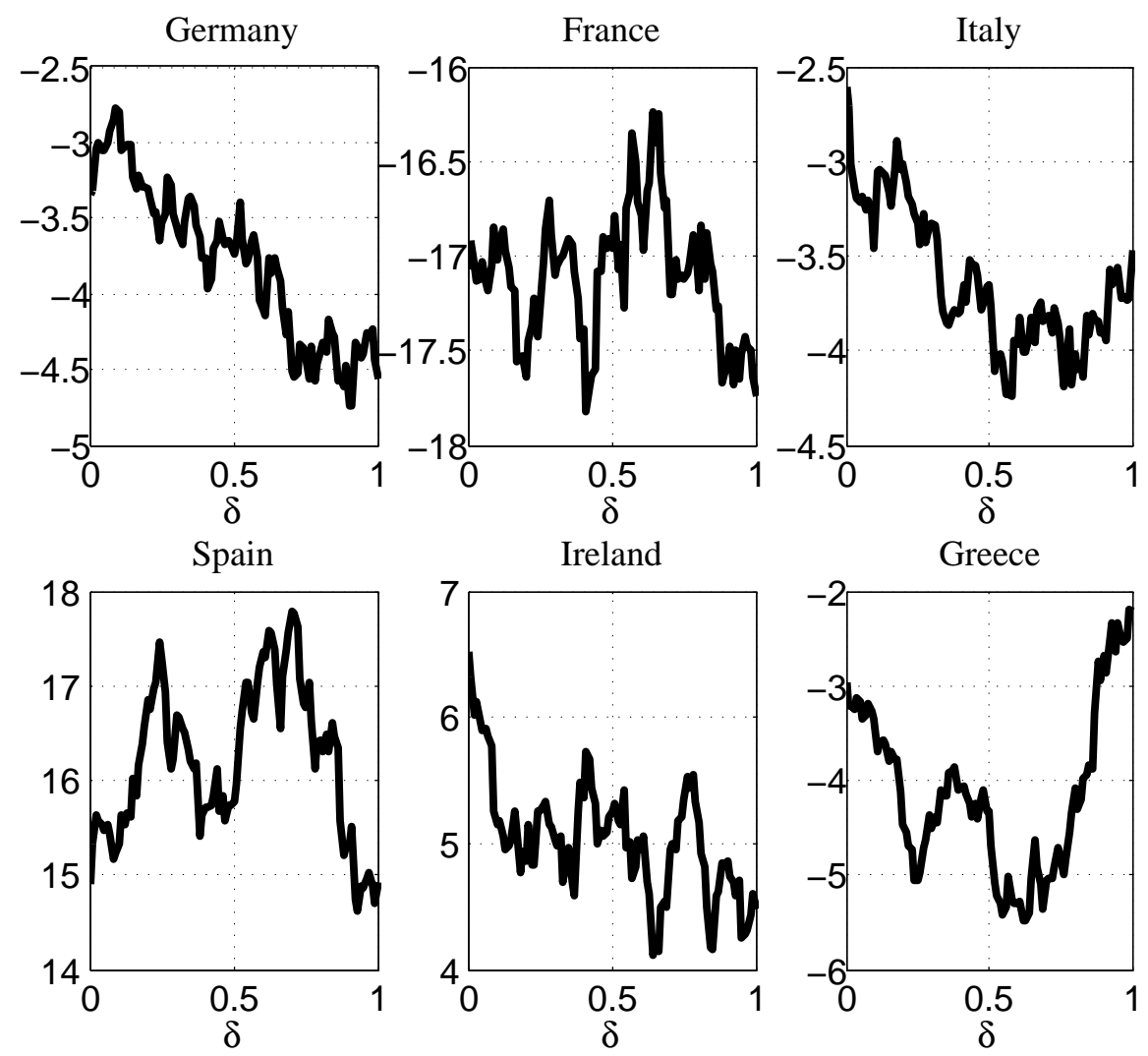

Figure 17: Contaminated mean-variance allocation as a function of $\delta$. 


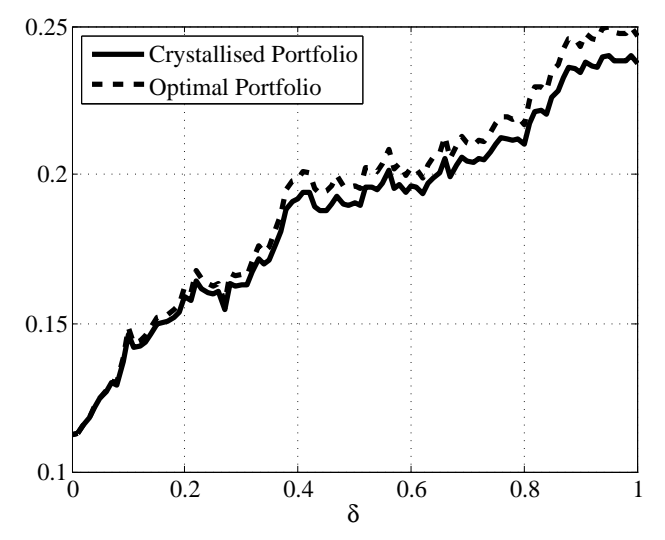

Figure 18: Impulse response of the Sharpe ratio of crystallized and Mean-Variance portfolios (contamination in distribution).
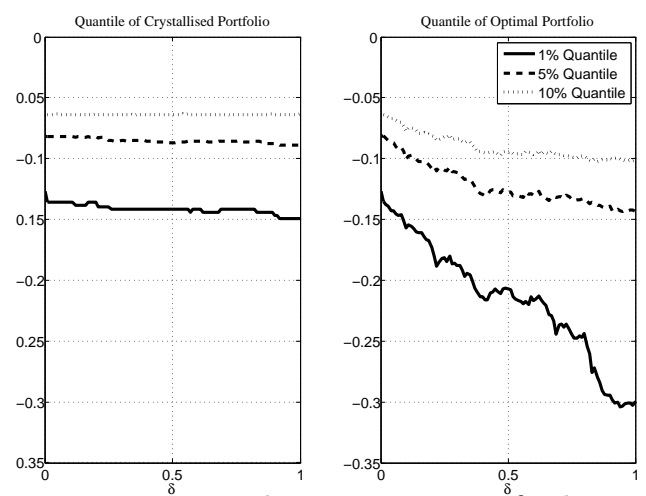

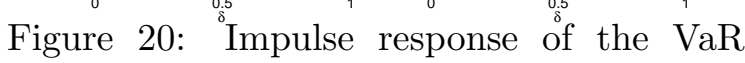
of crystallized and Mean-Variance portfolios (contamination in distribution).
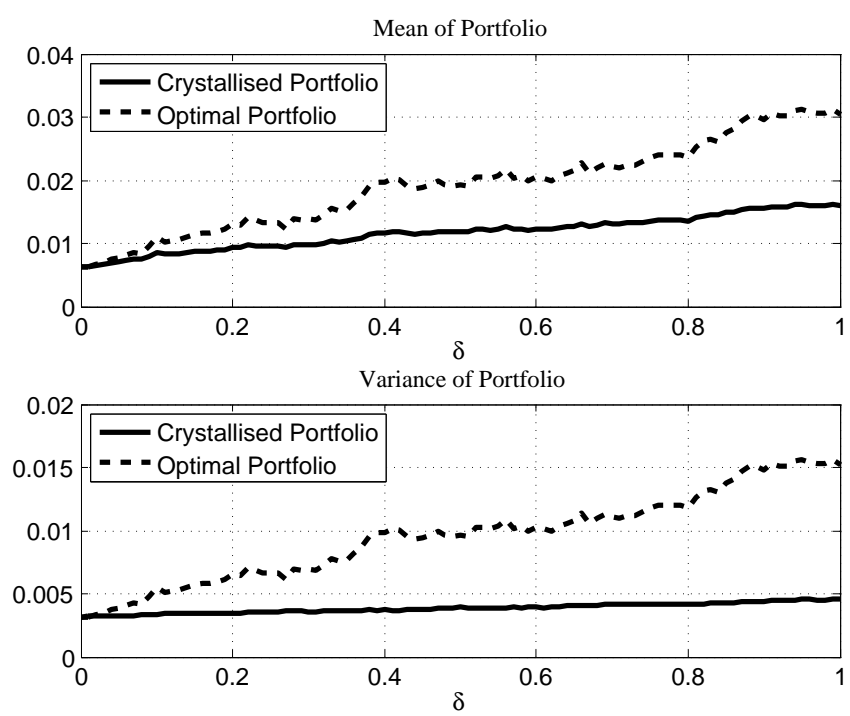

Figure 19: Impulse response of the Mean and Variance of crystallized and Mean-Variance portfolios (contamination in distribution).
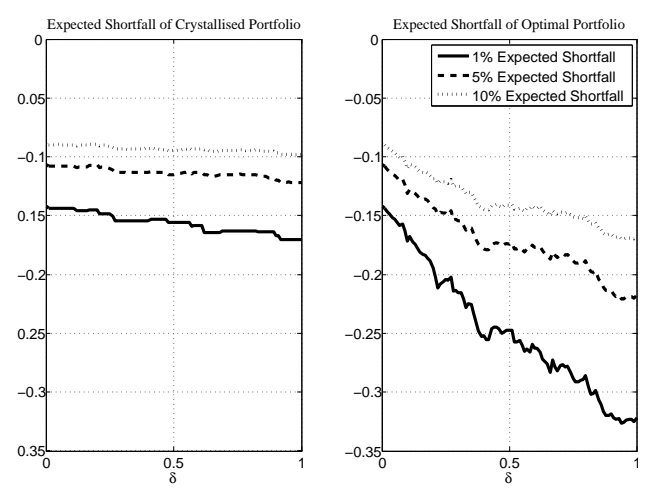

Figure 21: Impulse response of the Expectedshortfall of crystallized and Mean-Variance portfolios (contamination in distribution). 